\title{
Emerging Antineoplastic Biogenic Gold Nanomaterials for Breast Cancer Therapeutics: A Systematic Review
}

This article was published in the following Dove Press journal: International Journal of Nanomedicine

\author{
Muthupandian Saravanan (D) \\ Hossein Vahidi ${ }^{2}$ \\ David Medina Cruz (iD) 3,4 \\ Ada Vernet-Crua (iD ${ }^{3,4}$ \\ Ebrahim Mostafavi (iD) 3,4 \\ Ryan Stelmach ${ }^{3}$ \\ Thomas J Webster (iD) 3,4 \\ Mohammad Ali Mahjoub (iD ${ }^{5}$ \\ Masoumeh Rashedi ${ }^{6}$ \\ Hamed Barabadi (iD) ${ }^{2}$ \\ 'Department of Medical Microbiology and \\ Immunology, Division of Biomedical Science, \\ School of Medicine, College of Health Sciences, \\ Mekelle University, Mekelle I87I, Ethiopia; \\ ${ }^{2}$ Department of Pharmaceutical Biotechnology, \\ School of Pharmacy, Shahid Beheshti University of \\ Medical Sciences, Tehran, Iran; ${ }^{3}$ Department of \\ Chemical Engineering, Northeastern University, \\ Boston, MA, USA; ${ }^{4}$ Nanomedicine Science and \\ Technology Center, Northeastern University, \\ Boston, MA, USA; ${ }^{5}$ Department of Pharmaceutics, \\ School of Pharmacy, Shahid Beheshti University of \\ Medical Sciences, Tehran, Iran; ${ }^{6}$ Student Research \\ Committee, School of Medicine, Gonabad \\ University of Medical Sciences, Gonabad, Iran
}

Correspondence: Thomas J Webster Department of Chemical Engineering, Nanomedicine Science and Technology Center, Northeastern University, Boston, MA, USA

Tel + I 6173736585

Email th.webster@neu.edu

Hamed Barabadi

Department of Pharmaceutical

Biotechnology, School of Pharmacy,

Shahid Beheshti University of Medical

Sciences, PO Box 14155-6153, Tehran

199195338I, Iran

$\mathrm{Tel} / \mathrm{Fax}+982 / 88665250$

Email barabadi@sbmu.ac.ir

\begin{abstract}
Breast cancer remains as a concerning global health issue, being the second leading cause of cancer deaths among women in the United States (US) in 2019. Therefore, there is an urgent and substantial need to explore novel strategies to combat breast cancer. A potential solution may come from the use of cancer nanotechnology, an innovative field of study which investigates the potential of nanomaterials for cancer diagnosis, therapy, and theranostic applications. Consequently, the theranostic functionality of cancer nanotechnology has been gaining much attention between scientists during the past few years and is growing exponentially. The use of biosynthesized gold nanoparticles (AuNPs) has been explored as an efficient mechanism for the treatment of breast cancer. The present study supposed a global systematic review to evaluate the effectiveness of biogenic AuNPs for the treatment of breast cancer and their anticancer molecular mechanisms through in vitro studies. Online electronic databases, including Cochrane, PubMed, Scopus, Web of Science, Science Direct, ProQuest, and Embase, were searched for the articles published up to July 16, 2019. Our findings revealed that plant-mediated synthesis was the most common approach for the generation of AuNPs. Most of the studies reported spherical or nearly spherical-shaped AuNPs with a mean diameter less than $100 \mathrm{~nm}$ in size. A significantly larger cytotoxicity was observed when the biogenic AuNPs were tested towards breast cancer cells compared to healthy cells. Moreover, biogenic AuNPs demonstrated significant synergistic activity in combination with other anticancer drugs through in vitro studies. Although we provided strong and comprehensive preliminary in vitro data, further in vivo investigations are required to show the reliability and efficacy of these NPs in animal models.
\end{abstract}

Keywords: cancer nanotechnology, nanotoxicity, gold nanoparticles, anti-cancer

\section{Introduction}

Cancer remains the second leading cause of death worldwide with an estimated of 606,880 annual deaths in the United States. The American Cancer Society (ACS) estimates that 1,762,450 new cases of cancer will be diagnosed in the US just in 2019 with more than 15 million US citizens carrying a history with cancer. ${ }^{1}$ Meanwhile, breast cancer remains the most frequent cancer in women, and a significant public health issue globally. ${ }^{2}$ Both of the developing and developed world are suffering from breast cancer incidence and mortality. ${ }^{3}$ Approximately 1.5 million new cases of breast cancer are reported each year, corresponding for $25 \%$ of all cancer cases worldwide. ${ }^{4}$ Breast cancer remains at the top of newly diagnosed cancer cases and is the second leading cause of cancer deaths among US females, accounting for $30 \%$ of all cancer cases and $15 \%$ of cancer deaths in 
women. For instance, data from the ACS anticipates 42,260 breast cancer deaths comprising 41,760 female and 500 male in the US in 2019. ${ }^{1}$ Most of these deaths will be related to failure in diagnosis and treatment, since several parameters, including tumor size, grade, invasion statue to lymph nodes, human epidermal growth factor receptor 2 (HER2), surgical margins or age of the patient, among others, have a significant impact on the handling of the disease. ${ }^{5}$

As happens with other cancer types, currently available breast cancer therapies are not valid for most patients. The lack of selective cytotoxicity to tumors, and as a consequence the adverse effects of current drugs, lead to a reduced therapeutic index, and subsequently increased number of patients who receive suboptimal drug doses. ${ }^{6}$ One pivotal factor involved in treatment failure is the specific cancer features. For solid tumors, high interstitial fluid pressure (IFP) acts as a barrier for the penetration of the drugs through the capillaries, ${ }^{7}$ while the overexpression of drug efflux proteins makes the tumor cells resistant to drugs and causes therapeutic failure. ${ }^{8-10}$ Moreover, the short half-life of some chemotherapy drugs also limits the ability of the compounds to efficiently penetrate the tumor tissue. $^{11}$

Consequently, the considerable global mortality of breast cancer, along with the economic burden placed on modern society, craves for the exploration of new strategies to combat breast cancer. Nanotechnology, as an emerging field with outstanding applications in medicine, might have an answer. Nanomedicine, as the interdisciplinary fusion of nanotechnology and medicine, is a field of study that offers new opportunities to combat cancer with entirely new and renovated approaches involving the use of nanostructures. The behavior of nanomaterials (NMs) is different from their counterparts in the bulk scale due to their large surface-to-volume ratio, transforming them in highly reactive materials. Their unique physicochemical properties provide a chance to design a new generation of cancer theranostic compounds, ${ }^{12-14}$ with a high degree of tunability, allowing scientist to produce NMs with different compositions, shapes, sizes and desired responses in the targeted biological systems. ${ }^{15}$

The application of nanotechnology to combat cancer has generated a new field of study which is named "Cancer Nanotechnology," described as the use of nanotechnology for cancer detection, diagnosis, imaging, and therapy. ${ }^{16}$ Nanoscale-based drugs provide considerable benefits over free drugs such as an increased selectivity, hence, decreasing the systemic toxicity and enhancing the penetration of drugs into targeted tissue, while preventing the compounds from undergoing early degradation. ${ }^{17}$ According to the literature, metallic nanoparticles (MNPs) have significant potential for biomedical applications, including cancer therapy. ${ }^{18}$ Consequently, MNPs are prospective candidates for the nextgeneration of anticancer drugs, ${ }^{19}$ which can be prepared via a facile and straightforward approach using different physicochemical approaches. ${ }^{20}$

Nevertheless, there is a constant rise of environmental concerns related to the production of NMs employing traditional nanotechnology, which is leading the scientific community to focus on the design and use of environmentallyfriendly, cost-effective and biocompatible raw materials and processes for the generation of these nanostructures. Green Nanotechnology, as an emerging field within nanotechnology, offers the possibility to produce NMs without the generation of toxic by-products or consumption of harsh chemicals for synthesis and the subsequent functionalization of NMs. Green-synthesized NMs are therefore suitable for biomedical applications, hence overcoming many limitations of traditional physicochemical approaches. Green nanotechnological approaches are characterized by the use of living organisms, such as bacteria, fungi, plants, biomolecules, and other natural raw materials for the efficient generation of NMs. ${ }^{21-35}$ Biomolecules that are extracted from natural sources are used as unique reducing and capping agents for the efficient reduction of metallic ions into differently-shaped elemental NMs, whose particular chemistry is undoubtedly linked to the properties of the raw material, hence producing a synergy between both materials. ${ }^{36,37}$ Among all possible chemistries employed for the production of MNPs, gold (Au) has reached the highest attention in terms of biomedical applications, with extensive research. As a consequence, gold nanoparticles (AuNPs) have become an interesting research area for cancer theranostics. ${ }^{19,38}$ Due to the unique physicochemical properties of AuNPs, they have been investigated for various applications related to cancer, such as gene therapy, ${ }^{39}$ targeted drug delivery, ${ }^{40,41}$ radiotherapy, ${ }^{42,43}$ tumor detection, ${ }^{44}$ and cellular Bioimaging. ${ }^{45}$

A previous study systematically reviewed the efficacy of biologically synthesized AuNPs against prostate cancer cells through in vitro models and reported their significant prostate anticancer activity. ${ }^{13}$ However, to the best of our knowledge, no comprehensive study has reviewed the efficacy of biogenic AuNPs against breast cancer cells. Hence, in the current article, we aimed to conduct a systematic review on published papers to evaluate the anticancer activity of biogenic AuNPs against breast cancer cells through in vitro models. At the end, an 
in vitro molecular mechanism of how biogenic AuNPs can act against breast cancer cells is proposed.

\section{Methods}

The present study is a systematic review by the guidelines of the Preferred Reporting Items for Systematic Reviews and Meta-Analyses (PRISMA) ${ }^{46}$ to evaluate the anticancer activity of biogenic AuNPs against breast cancer cells through in vitro investigations. The research was followed by an iterative process comprehended a search strategy and a study selection, with an inclusion and exclusion criteria that allowed for an efficient data collection.

\section{Search Strategy}

The guidelines of PRISMA were followed for the search strategy of the current study. ${ }^{46}$ The international electronic databases comprising Cochrane, Scopus, PubMed, Web of Science, Science Direct, ProQuest, and Embase were searched for the articles published up to July 16, 2019. The asterisk wildcard character $(*)$ was used to include alternative forms of words, plurals, etc. The keywords included "gold", "Au", and "synthesis", "fabrication", "biosynthesis", "biofabrication", "green", "bioreduction", "myco*", "biogenic", "biomimetic", "plant*", "phyto*”, "herbal", "fungal", "bacterial", "alga*", "microbial", "biological", and "nanoparticle*", "colloidal", "nanomaterial*", "nanostructure*”, "nano-gold", and "antitumor*", "anticancer*”, "antineoplastic", "cell line*", "cancer*", "tumor*", "cytotoxicity", "cytotoxic", as well as "breast".

\section{Study Selection}

The eligibility of the records was assessed by two independent researchers through first screening (reviewing of the titles and abstracts) and second screening (reviewing full texts). A third investigator judged for any disagreement.

\section{Inclusion Criteria}

The eligible studies that met the following characteristics were included in the current study: a) papers identified from the aforementioned key search; b) peer-reviewed original research papers; c) papers including sufficient information; d) papers written in English; e) published and/or in press papers; f) papers that studied the anticancer activity of biogenic AuNPs against breast cancer cells through in vitro models; and g) papers that evaluated the biocompatibility of biogenic AuNPs with healthy breast cells through in vitro models.

\section{Exclusion Criteria}

Ineligible studies that met the following characteristics were excluded from the current study: a) duplicated records; b) non-related records; c) congress abstracts; d) non-laboratory studies such as editorials, review papers, case reports, and letters to the editors; e) non-English papers; and f) papers that did not study the anticancer activity of biogenic AuNPs against breast cancer cell lines.

\section{Data Collection}

A data extraction form (Table 1) was used to extract the following variables comprising first author, year of publication, a biological source with scientific name, characterization techniques, size $(\mathrm{nm})$, morphology, breast cancer cell line, standard cell line, dose, exposure time, cytotoxicity method, and significant outcome. Data extraction was performed by two researchers. A third researcher checked the data extraction forms. Any disagreement was resolved by discussion and consensus. Remarkably, through the articles that evaluated the cytotoxicity of breast cancer and healthy cells, we extracted the biogenic AuNPs-induced cytotoxicity data of other regular cell lines to compare the results.

\section{Results \\ Search Results}

Figure 1 depicts a PRISMA flowchart representing the results of our systematic search as well as the process of study selection. Briefly, of 1644 identified records identified through our systematic searches, 701 records were duplicate. The first screening was performed on 943 articles leading to the excluding of 858 articles according to the inclusion and exclusion criteria. Further, the second screening was conducted on 85 articles, and finally, 53 articles were found eligible to be included in the current systematic review.

\section{Characteristics of Included Studies}

Table 1 represents the results of cytotoxicity of biologically synthesized AuNPs against breast cancer cells as well as their biocompatibility data when cultured with normal cells. Of a total of fifty-three articles $(n=53)$, forty-two articles $(n=42)$ reported a plant-mediated synthesis process for the preparation of AuNPs in their studies. Hence, phytosynthesis of AuNPs was the most common approach (79.24\% of all studies) for the fabrication of AuNPs. Other studies (20.76\% of all studies) used bacteria $(\mathrm{n}=4)$, fungi $(\mathrm{n}=4)$, and algae $(\mathrm{n}=3)$ for the green synthesis of AuNPs as unique reducing and capping agents. Most of the reports showed the production 


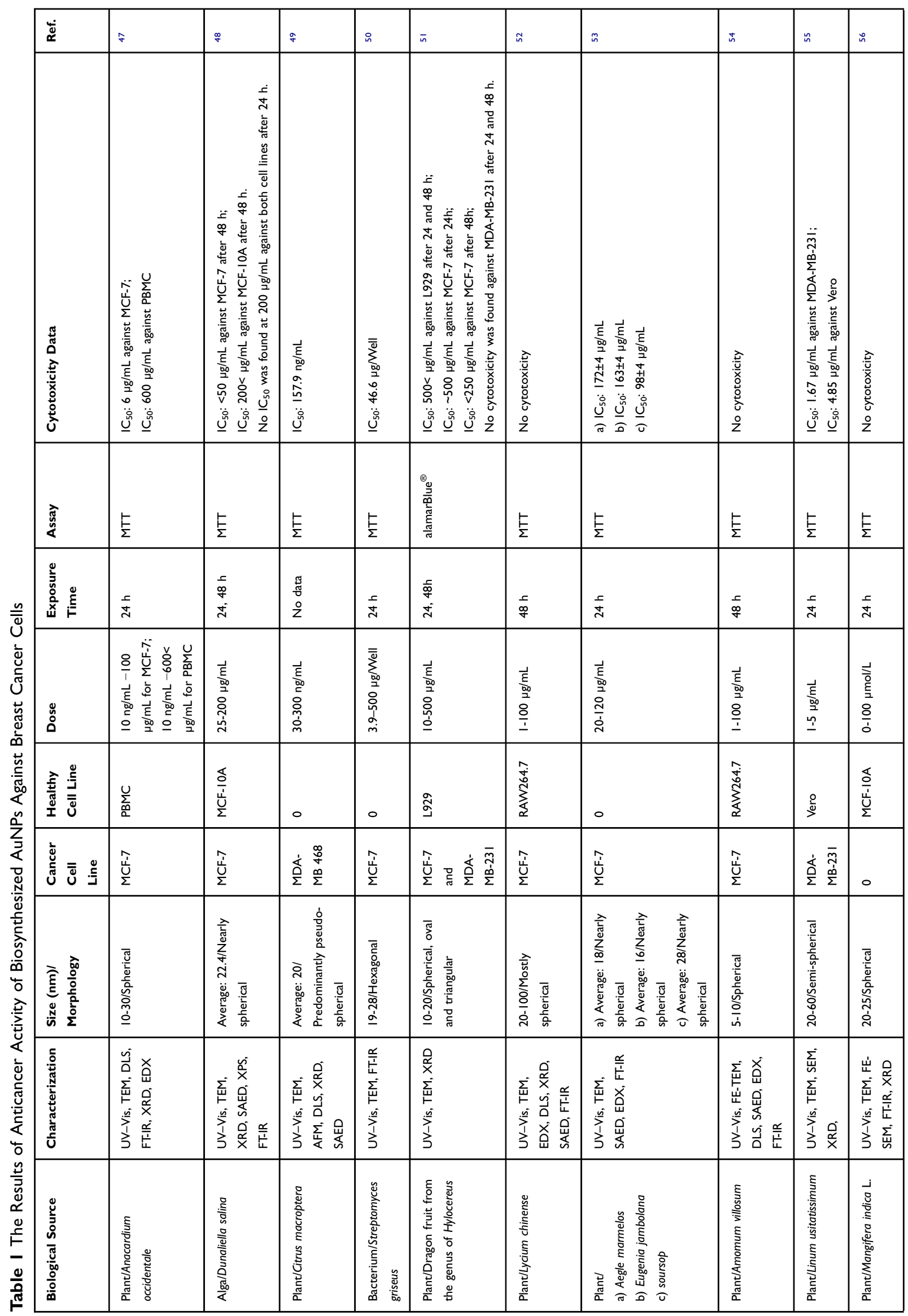




\begin{tabular}{|c|c|c|c|c|c|c|c|c|c|c|c|}
\hline in & $\stackrel{\infty}{n}$ & in & : & $\overline{0}$ & ธ & ๓⿴囗十 & t & $\ddot{0}$ & $\because$ & 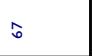 & œ \\
\hline 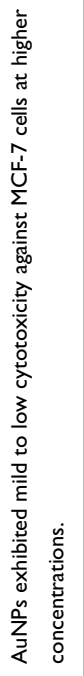 & 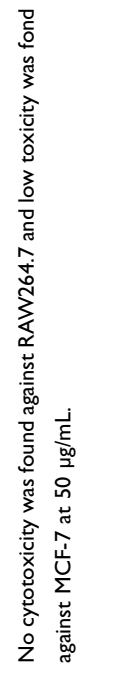 & 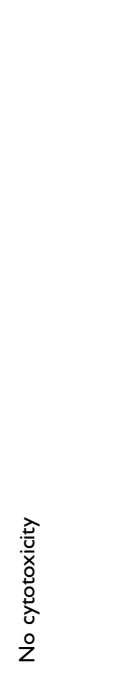 & 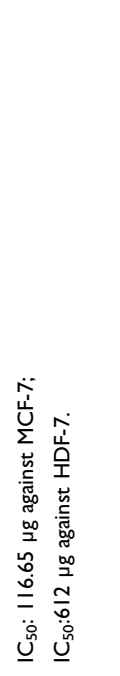 & 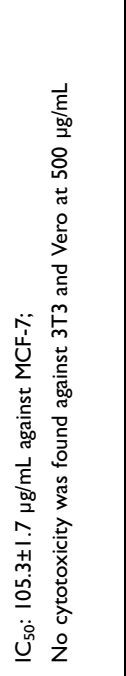 & 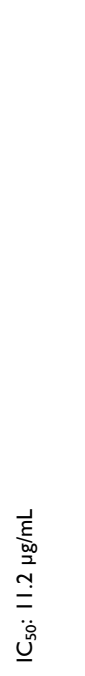 & 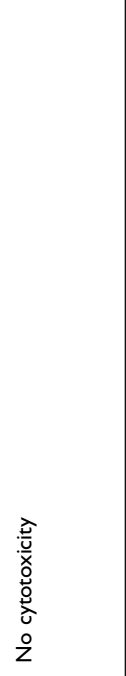 & 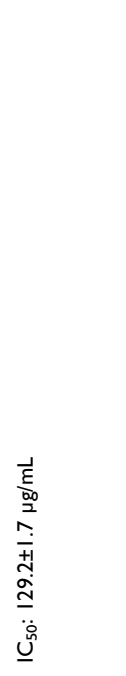 & 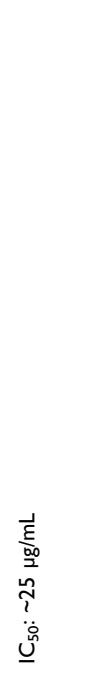 & 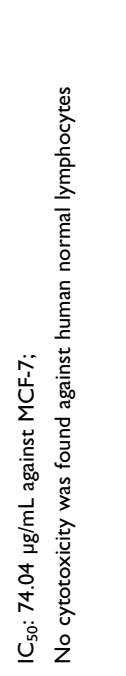 & 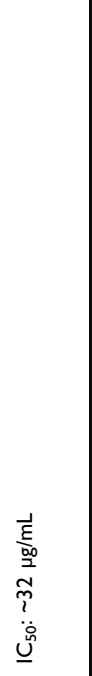 & 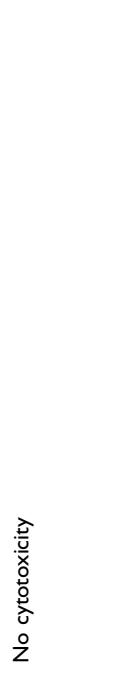 \\
\hline 卢 & $\frac{E}{\Sigma}$ & $\frac{\tilde{E}}{\Sigma}$ & $\frac{E}{\Sigma}$ & 占 & $\frac{E}{\Sigma}$ & 占 & $\begin{array}{l}\frac{\bar{E}}{\overline{0}} \\
\frac{\bar{y}}{\bar{n}}\end{array}$ & $\frac{E}{\Sigma}$ & $\frac{E}{\Sigma}$ & $\stackrel{5}{\xi}$ & 点 \\
\hline$\stackrel{d}{ \pm}$ & $\underset{\stackrel{c}{q}}{\stackrel{c}{q}}$ & $\underset{\stackrel{c}{\infty}}{\stackrel{c}{\sigma}}$ & $\stackrel{5}{ \pm}$ & $\frac{5}{d}$ & $\stackrel{5}{ \pm}$ & $\underset{\stackrel{s}{q}}{\stackrel{s}{q}}$ & $\underset{N}{\stackrel{5}{N}}$ & $\underset{\stackrel{c}{q}}{\stackrel{\infty}{q}}$ & 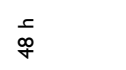 & $\stackrel{5}{ \pm}$ & $\underset{\text { q }}{\stackrel{5}{f}}$ \\
\hline 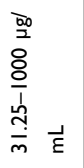 & 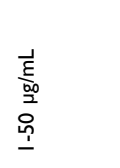 & 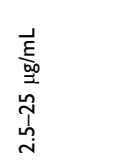 & $\begin{array}{l}\frac{00}{0} \\
\text { o } \\
\frac{0}{1} \\
\text { in }\end{array}$ & 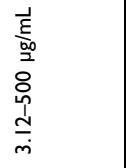 & 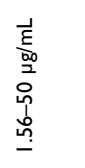 & 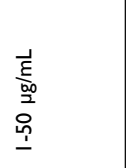 & 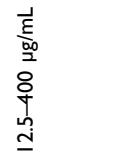 & $\begin{array}{l}\overrightarrow{\vec{\varepsilon}} \\
\frac{\underline{\underline{0}}}{20} \\
\frac{\delta}{\dot{d}} \\
\underline{\dot{d}}\end{array}$ & 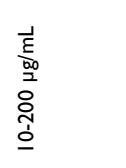 & 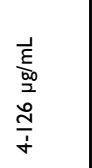 & $\begin{array}{l}\vec{k} \\
\frac{\underline{0}}{20} \\
\frac{0}{1} \\
\frac{1}{0} \\
0\end{array}$ \\
\hline ○ & 守 & 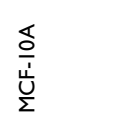 & 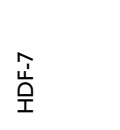 & 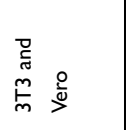 & ○ & 竎 & ○ & 0 & 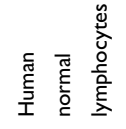 & 0 & 寄 \\
\hline 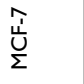 & $\frac{\hat{U}}{\Sigma}$ & 0 & $\begin{array}{l}\hat{U} \\
\dot{U}\end{array}$ & $\frac{\hat{U}}{\Sigma}$ & $\frac{\hat{U}}{\Sigma}$ & $\frac{\hat{U}}{\Sigma}$ & 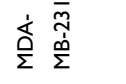 & 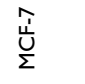 & $\frac{\widehat{U}}{\Sigma}$ & 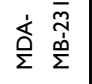 & $\frac{\tilde{U}}{\Sigma}$ \\
\hline 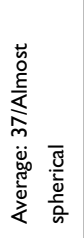 & 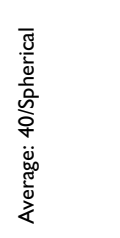 & 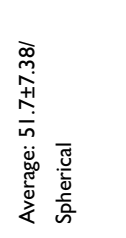 & 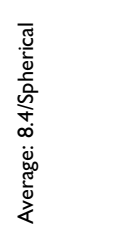 & 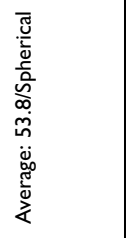 & 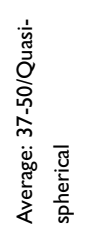 & 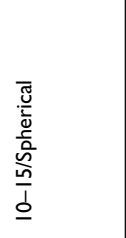 & 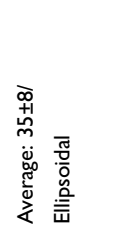 & 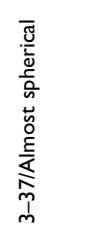 & 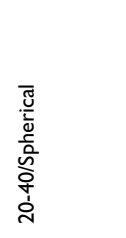 & 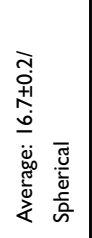 & 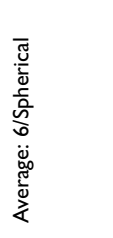 \\
\hline 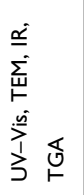 & 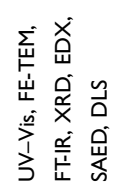 & 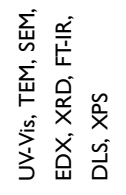 & 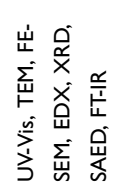 & 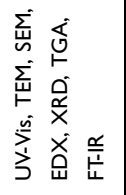 & 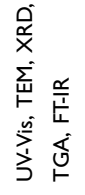 & 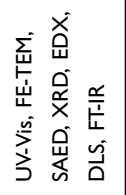 & 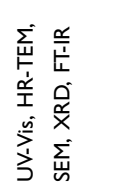 & 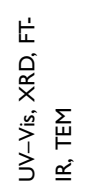 & 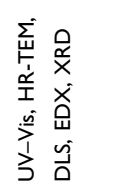 & 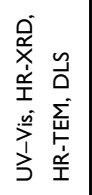 & 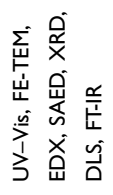 \\
\hline 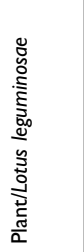 & 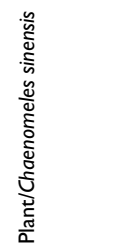 & 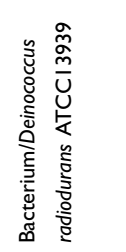 & 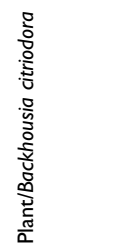 & 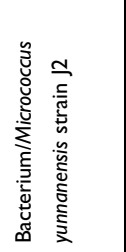 & 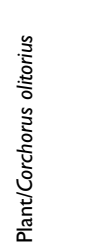 & 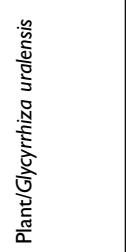 & 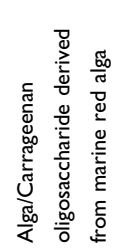 & 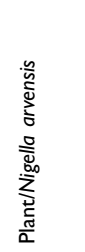 & 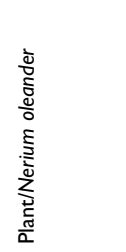 & 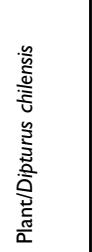 & 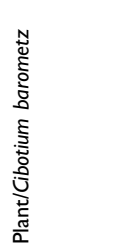 \\
\hline
\end{tabular}




\begin{tabular}{|c|c|c|c|c|c|c|c|c|}
\hline$\stackrel{\dot{\sigma}}{\mathscr{\alpha}}$ & \& & $\therefore$ & $\pi$ & ה & n & \pm & $n$ & $\therefore$ \\
\hline 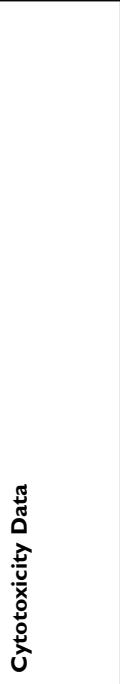 & 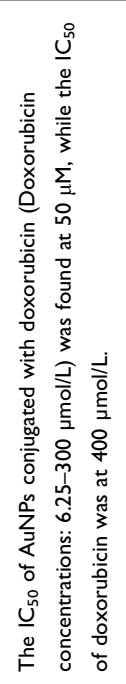 & 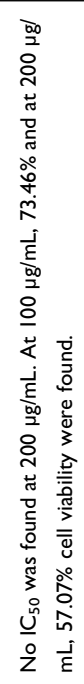 & 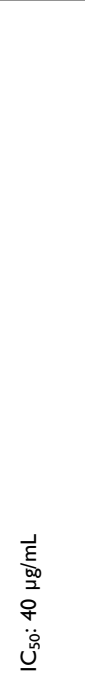 & 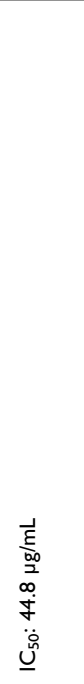 & 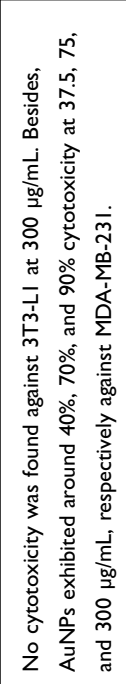 & 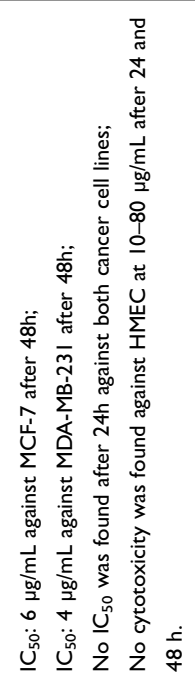 & 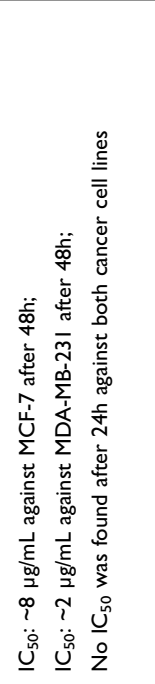 & 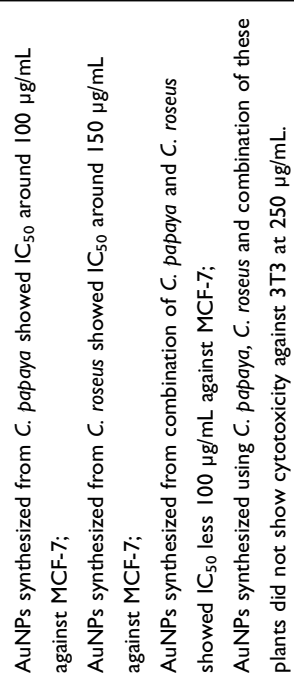 \\
\hline 鸽 & $\frac{E}{\Sigma}$ & $\frac{E}{\Sigma}$ & E & $\frac{E}{\Sigma}$ & E & $\sum$ & $\frac{F}{\Sigma}$ & E \\
\hline 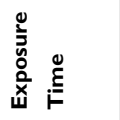 & 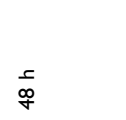 & $\stackrel{\nwarrow}{d}$ & 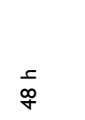 & 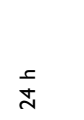 & $\mid$\begin{tabular}{|c}
$r$ \\
og
\end{tabular} & 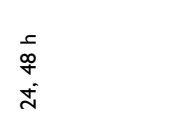 & $\begin{array}{l}\stackrel{5}{\infty} \\
\dot{\infty} \\
\dot{\sim}\end{array}$ & $\frac{5}{d}$ \\
\hline هั & 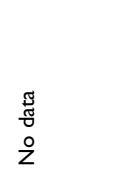 & 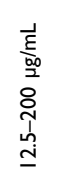 & $\begin{array}{l}\overrightarrow{\underline{E}} \\
\frac{\underline{0}}{2} \\
\underline{\underline{o}} \\
\underline{d}\end{array}$ & $\begin{array}{l}\vec{I} \\
\frac{\underline{g}}{\mathrm{wo}} \\
\frac{8}{I} \\
\frac{O}{I}\end{array}$ & 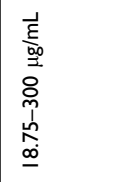 & 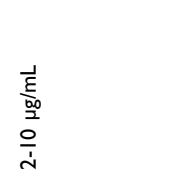 & 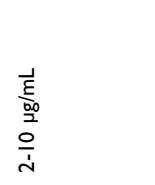 & 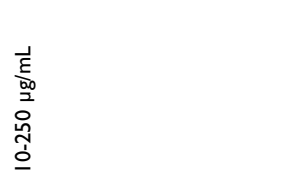 \\
\hline 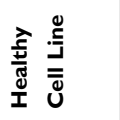 & 。 & o & 0 & 0 & $\overline{\overline{\dot{m}}}$ & $\underset{\frac{\underset{I}{I}}{\mathrm{U}}}{\mathrm{u}}$ & 。 & $\stackrel{m}{m}$ \\
\hline 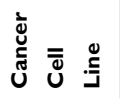 & 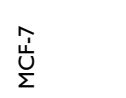 & $\frac{\widehat{U}}{\Sigma}$ & $\frac{\widehat{U}}{\Sigma}$ & $\frac{\hat{U}}{\Sigma}$ & 市 & 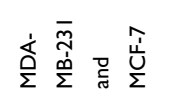 & 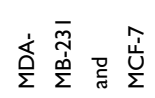 & $\frac{\hat{U}}{\frac{\hat{U}}{\Sigma}}$ \\
\hline 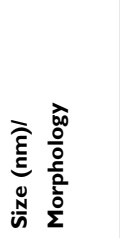 & 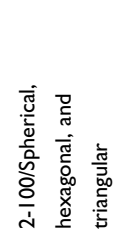 & 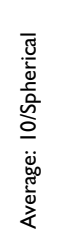 & 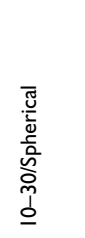 & 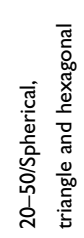 & 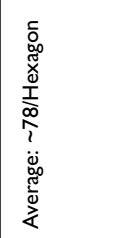 & 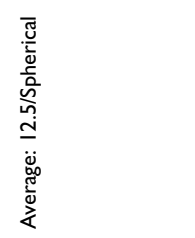 & 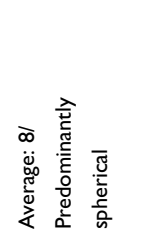 & 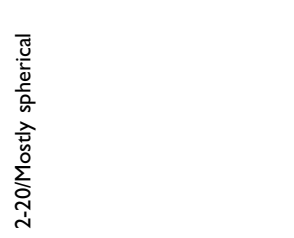 \\
\hline 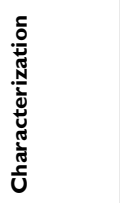 & 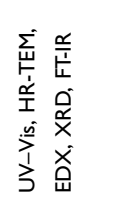 & 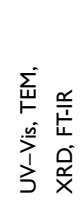 & 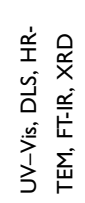 & 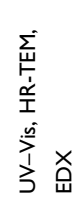 & 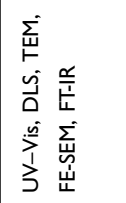 & 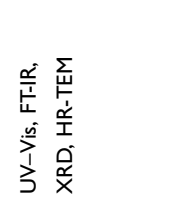 & 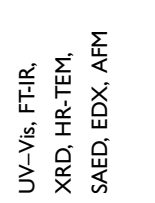 & 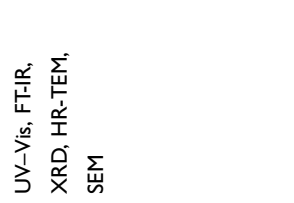 \\
\hline 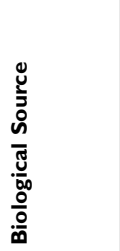 & 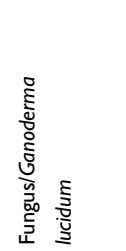 & 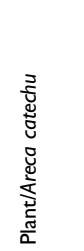 & 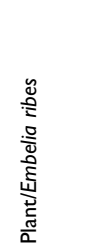 & 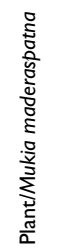 & 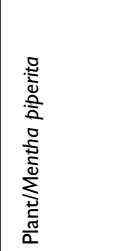 & 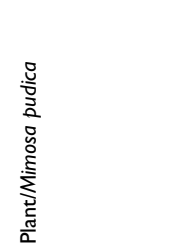 & 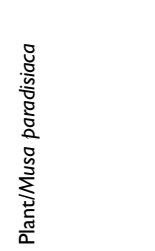 & 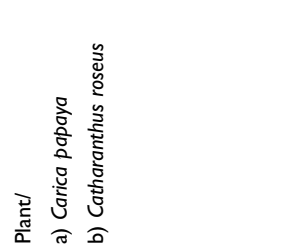 \\
\hline
\end{tabular}




\begin{tabular}{|c|c|c|c|c|c|c|c|c|c|c|}
\hline R & $\stackrel{\infty}{\curvearrowright}$ & $\approx$ & $\therefore$ & $\bar{\Phi}$ & $\approx$ & $\approx$ & Ф & 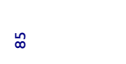 & × & ळิ \\
\hline 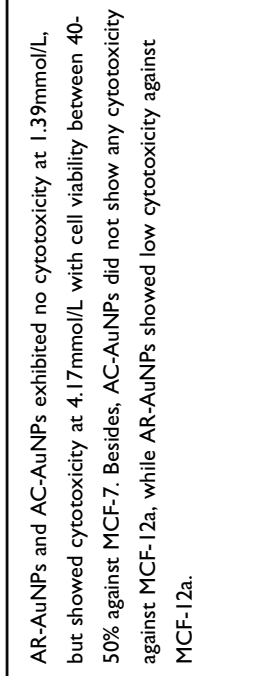 & 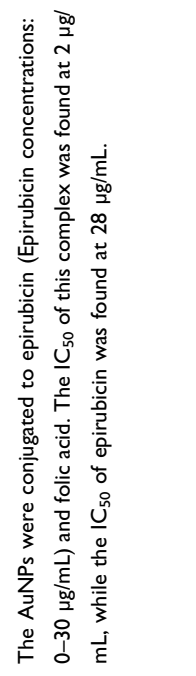 & 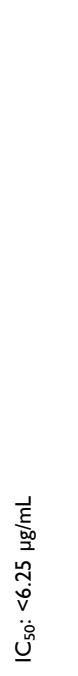 & 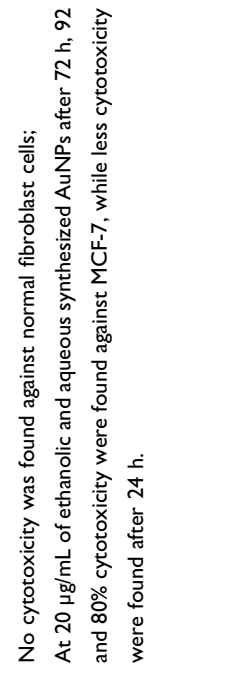 & 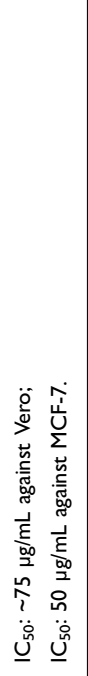 & 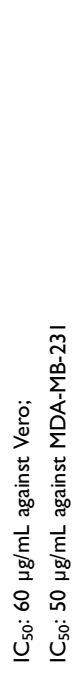 & $\begin{array}{l}\overrightarrow{\underline{\varepsilon}} \\
\underline{\text { bo }} \\
\underline{0} \\
\underline{\dot{\hat{\theta}}} \\
\underline{\underline{u}}\end{array}$ & 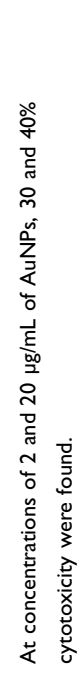 & 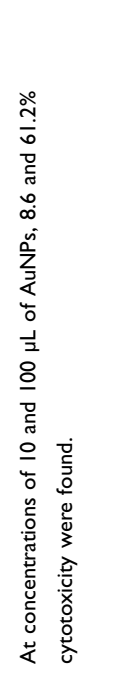 & 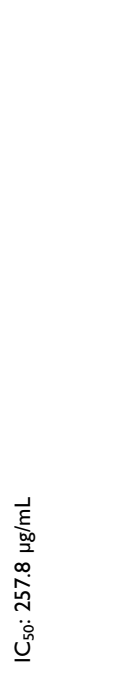 & 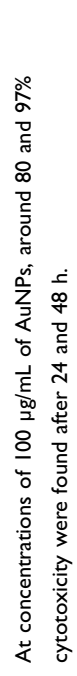 \\
\hline$E$ & $\frac{E}{\Sigma}$ & $\frac{5}{\Sigma}$ & $\frac{E}{\Sigma}$ & $\frac{E}{\Sigma}$ & 卢 & $\frac{E}{\Sigma}$ & $\frac{E}{\Sigma}$ & $\stackrel{5}{3}$ & 昼 & $\frac{E}{\Sigma}$ \\
\hline$\stackrel{5}{ \pm}$ & 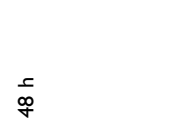 & 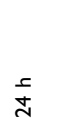 & 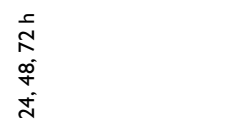 & $\stackrel{5}{ \pm}$ & $\stackrel{5}{\stackrel{s}{q}}$ & $\stackrel{s}{t}$ & $\stackrel{5}{ \pm}$ & $\stackrel{5}{ \pm}$ & $\underset{\stackrel{f}{q}}{\stackrel{5}{2}}$ & 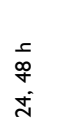 \\
\hline 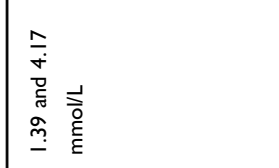 & 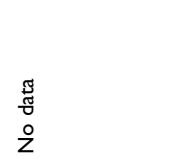 & 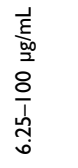 & $\begin{array}{l}\overrightarrow{\vec{E}} \\
\text { cog } \\
\text { ì } \\
\text { ì }\end{array}$ & $\begin{array}{l}\overrightarrow{\underline{E}} \\
\text { wo } \\
\frac{8}{\dot{b}} \\
\frac{8}{n}\end{array}$ & 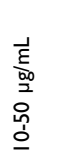 & 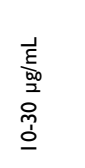 & 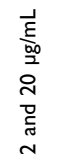 & 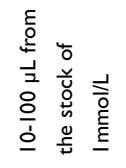 & 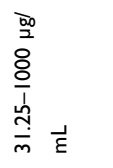 & 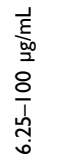 \\
\hline 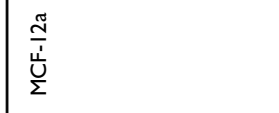 & 。 & 0 & 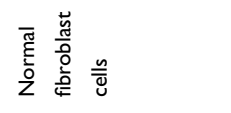 & $\stackrel{\frac{9}{\nu}}{\stackrel{2}{\nu}}$ & 。 & 0 & 0 & 0 & 。 & 。 \\
\hline 崩 & $\frac{\hat{U}}{\Sigma}$ & $\frac{\hat{U}}{\Sigma}$ & $\frac{\hat{U}}{\Sigma}$ & 岁 & 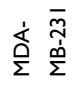 & $\stackrel{\dot{d}}{\Sigma} \frac{\infty}{\Sigma}$ & $\frac{\widehat{U}}{\Sigma}$ & $\frac{\widehat{U}}{\Sigma}$ & $\frac{\hat{U}}{\Sigma}$ & $\frac{\hat{U}}{\Sigma}$ \\
\hline 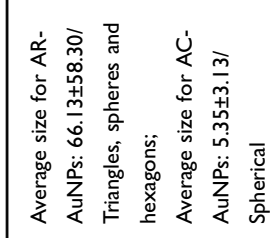 & 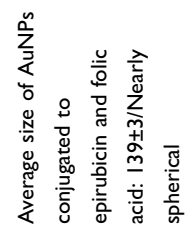 & 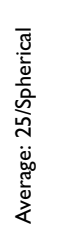 & 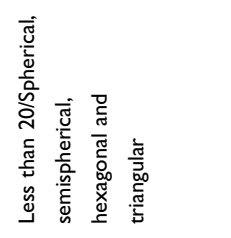 & 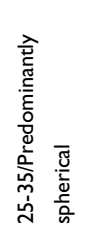 & 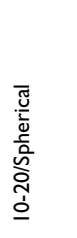 & 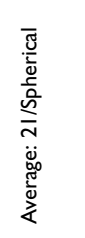 & 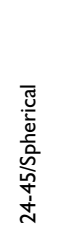 & 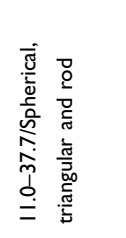 & 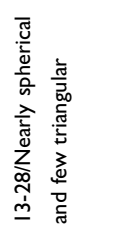 & 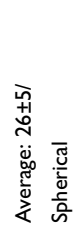 \\
\hline 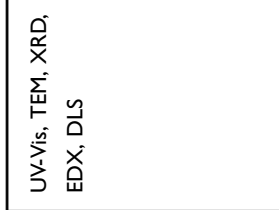 & 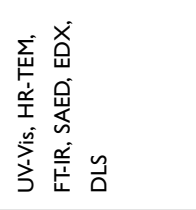 & 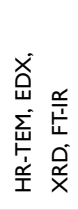 & 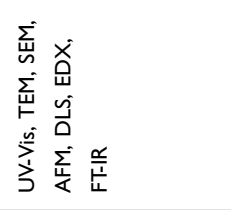 & 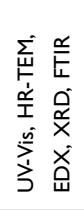 & 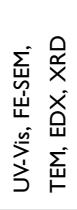 & 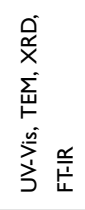 & 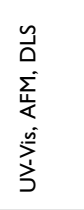 & 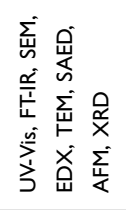 & 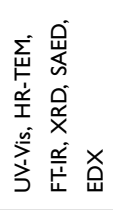 & 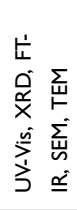 \\
\hline 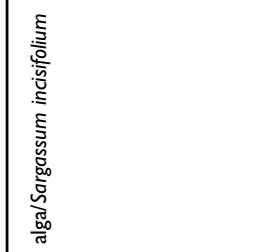 & 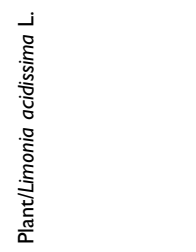 & 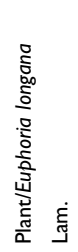 & 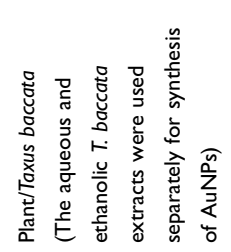 & 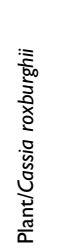 & 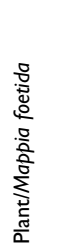 & 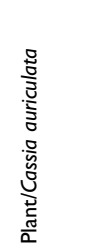 & 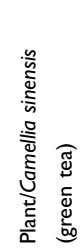 & 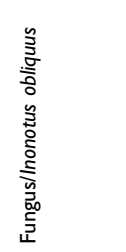 & 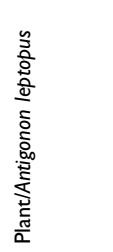 & 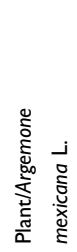 \\
\hline
\end{tabular}




\begin{tabular}{|c|c|c|c|c|c|c|c|c|c|c|c|}
\hline$\ddot{\mathscr{\Phi}}$ & $\infty$ & $\infty$ & $\therefore$ & $\bar{a}$ & $\approx$ & $\approx$ & ذ & ڤ & $\approx$ & $\approx$ & \& \\
\hline 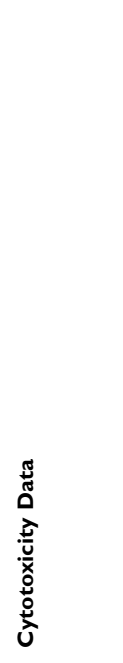 & 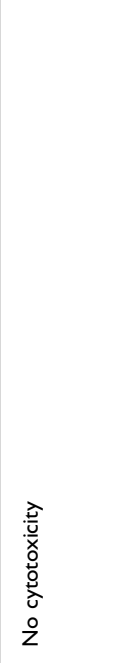 & 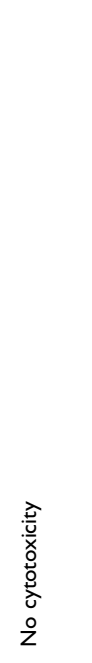 & 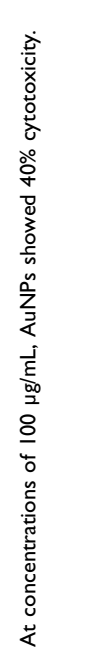 & 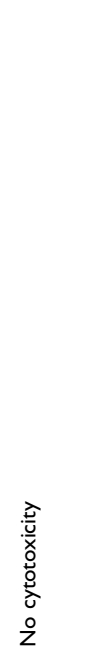 & 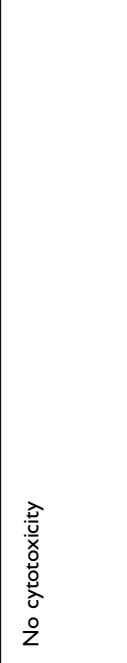 & 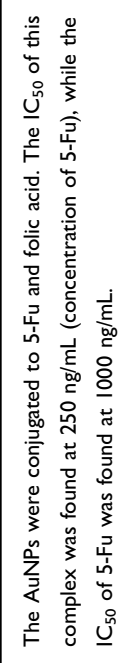 & 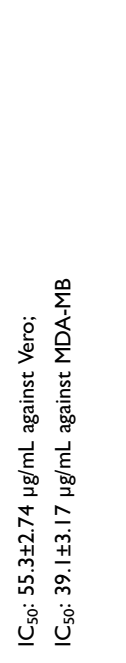 & 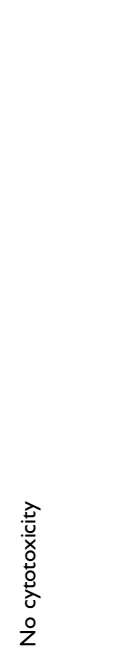 & 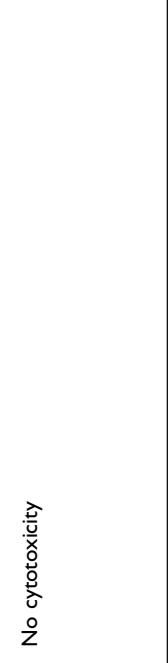 & 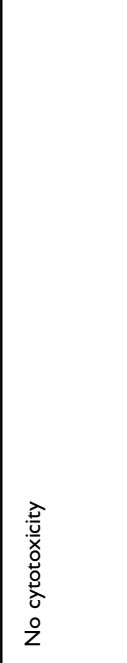 & 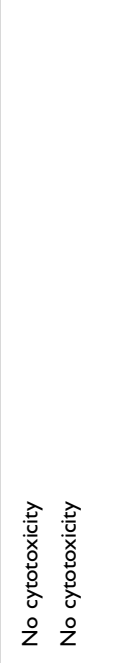 \\
\hline 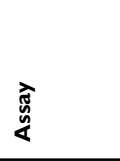 & $\frac{E}{\Sigma}$ & $\frac{5}{\Sigma}$ & $\frac{E}{\Sigma}$ & $\frac{E}{\Sigma}$ & 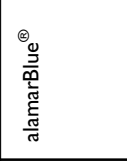 & $\frac{E}{\Sigma}$ & $\frac{E}{\Sigma}$ & $\frac{E}{\Sigma}$ & $\frac{E}{\Sigma}$ & 占 & $\frac{E}{\Sigma} \frac{E}{\Sigma}$ \\
\hline 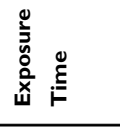 & 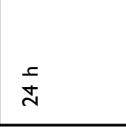 & $\underset{\substack{c \\
\stackrel{\infty}{+}}}{ }$ & 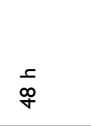 & $\stackrel{\tau}{\dot{d}}$ & $\frac{d}{4}$ & 辛 & $\stackrel{5}{i}$ & $\stackrel{5}{\overleftarrow{d}}$ & 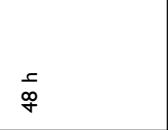 & 亲 & $\frac{5}{ \pm}$ \\
\hline ڤั & 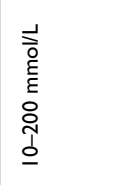 & $\begin{array}{l}\vec{a} \\
\vdots \\
\frac{n}{n} \\
\dot{\omega}\end{array}$ & 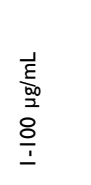 & $\begin{array}{l}\frac{1}{0} \\
\frac{\xi_{2}}{2} \\
\frac{8}{1} \\
\frac{8}{1}\end{array}$ & 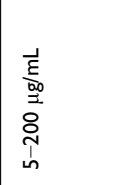 & 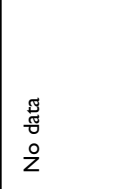 & 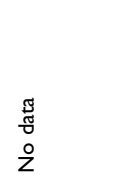 & 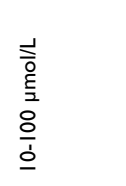 & 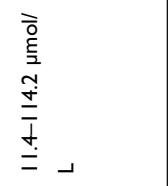 & 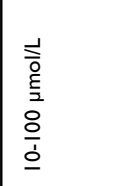 & 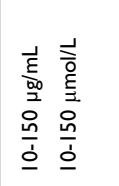 \\
\hline 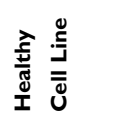 & 0 & ఫิ & 0 & 0 & 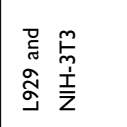 & 0 & $\stackrel{\circ}{\stackrel{\circ}{\nu}}$ & 。 & 0 & 0 & $\begin{array}{l}\frac{8}{\dot{1}} \\
\text { 产 }\end{array}$ \\
\hline 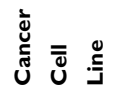 & $\frac{\hat{U}}{\Sigma}$ & $\underset{⿱ \stackrel{f}{f}}{\stackrel{\rho}{r}}$ & 离 & 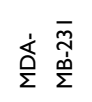 & 市 & \begin{tabular}{|l}
$\hat{U}$ \\
$\Sigma$
\end{tabular} & 暠 & $\frac{\widehat{U}}{\Sigma}$ & 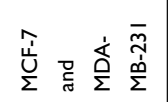 & $\frac{\widehat{U}}{\Sigma}$ & 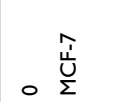 \\
\hline 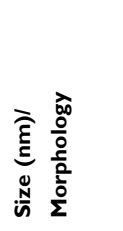 & 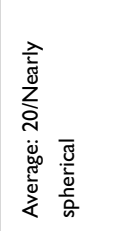 & 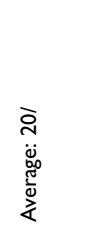 & 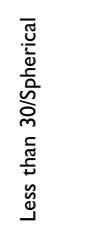 & 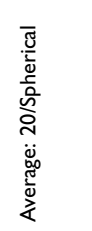 & 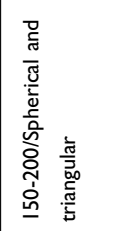 & 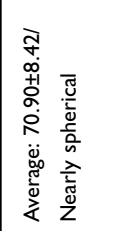 & 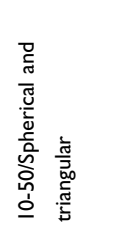 & 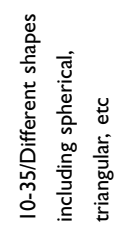 & 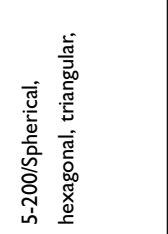 & 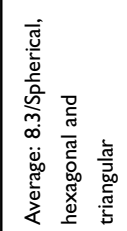 & 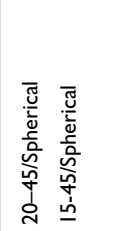 \\
\hline 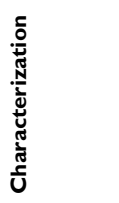 & 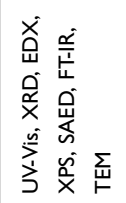 & 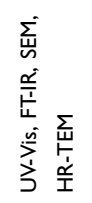 & 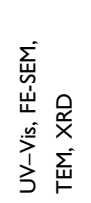 & 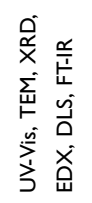 & 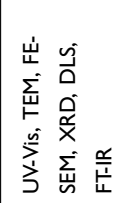 & 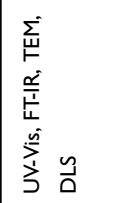 & 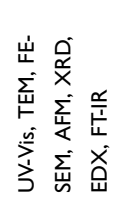 & 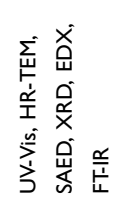 & 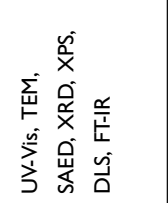 & 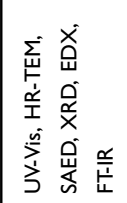 & 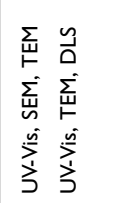 \\
\hline 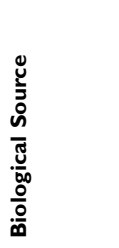 & 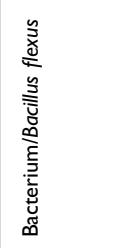 & 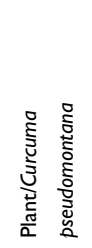 & 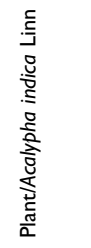 & 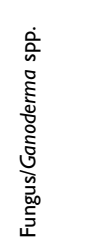 & 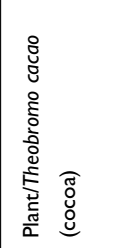 & 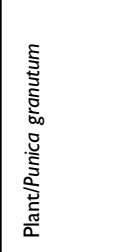 & 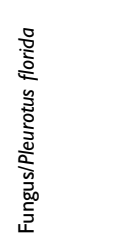 & 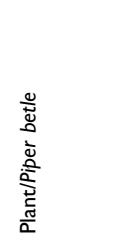 & 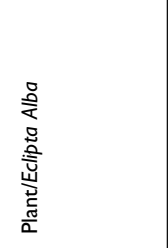 & 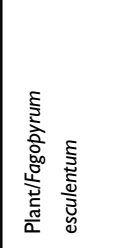 & 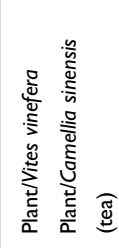 \\
\hline
\end{tabular}




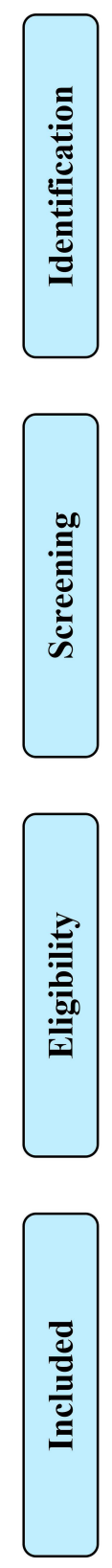

Figure I The PRISMA flowchart used in this study.

of spherical or nearly spherical shaped NPs with a mean diameter size less than $100 \mathrm{~nm}$. The cytotoxicity of AuNPs was assessed using five cytotoxic methods including MTT (3-(4,5-dimethylthiazol-2-yl)-2,5-diphenyltetrazolium bromide), MTS (3-(4,5-dimethylthiazol-2-yl)-5(3-carboxymethonyphenol)-2-(4-sulfophenyl)-2H-tetrazolium), WST (2-(4-iodophenyl)-3-(4-nitrophenyl)-5-(2,4-disulfophenyl)$2 \mathrm{H}$-tetrazolium), alamarBlue ${ }^{\circledR}$ (a weakly fluorescent blue indicator dye called resazurin), and Sulforhodamine B (2-(3-diethylamino-6-diethylazaniumylidene-xanthan -9-yl)-5-sulfo-benzenesulfonate) assays. The anticancer activity of AuNPs was assessed against four breast cancer cells, including MDA-MB-468, MCF-7, MDA-MB, and MDA-MB-231 (human breast adenocarcinoma cell), and T47D (human breast ductal carcinoma). Among these breast cancer cell lines, MCF-7 was the predominant cell line that was used for the evaluation of anti-breast cancer activity of AuNPs ( $\mathrm{n}=38$ ), while the second place belongs to MDA-MB -231 cells $(n=12)$. Furthermore, although several studies reported less or no cytotoxicity of biosynthesized AuNPs against breast cancer cells, a number of examples showed a half-maximal inhibitory concentration $\left(\mathrm{IC}_{50}\right)$ representing 

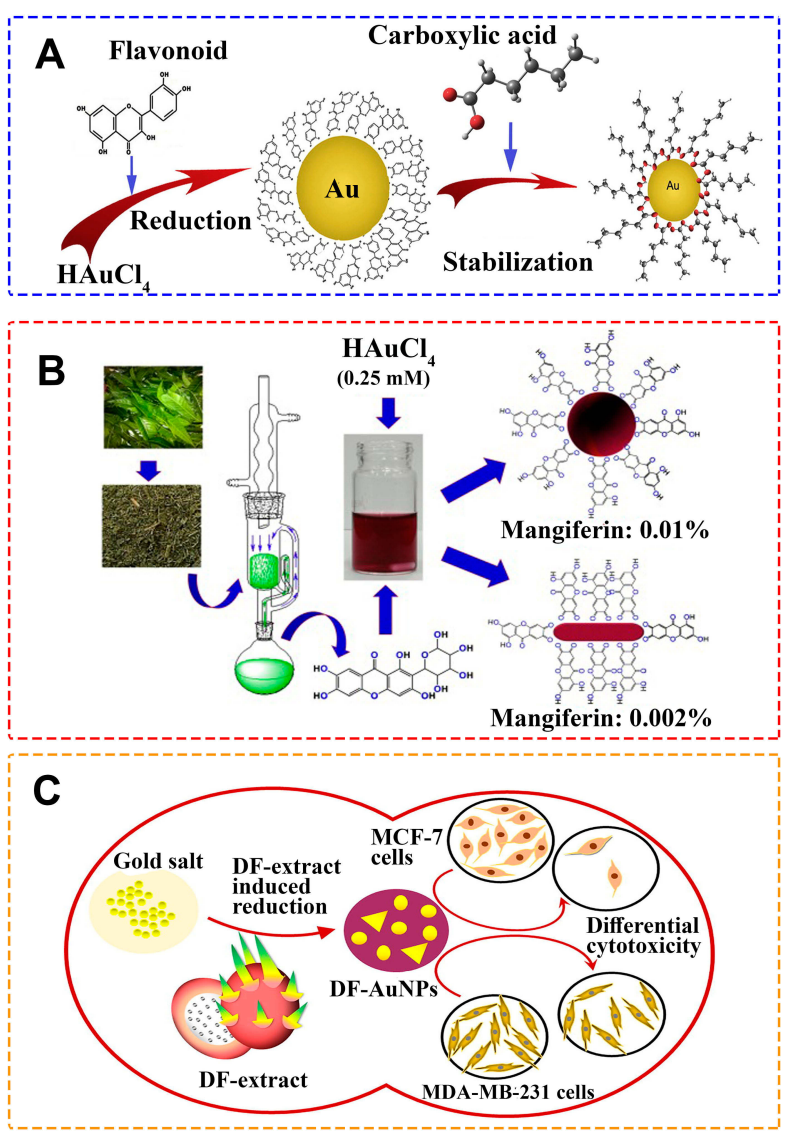

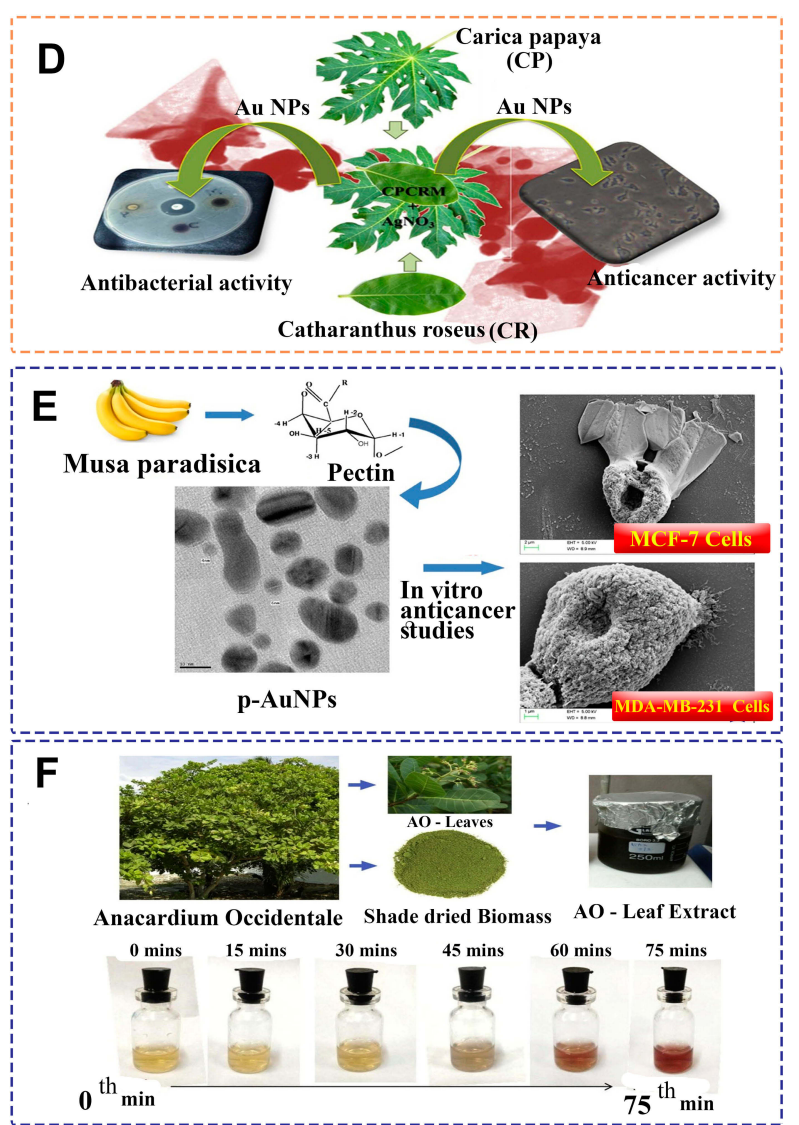

Figure 2 Schematic visualization of reduction and stabilization of AuNPs using different natural sources. (A) Schematic visualization of reduction and stabilization of AuNPs using different fruit extracts. Reprinted from J Saudi Chem Soc. Vol 23. Vijayakumar S. Eco-friendly synthesis of gold nanoparticles using fruit extracts and in vitro anticancer studies, pages 753-76I, Copyright 2019, with permission from Elsevier. ${ }^{53}$ (B) Synthetic outline for the isolation of mangiferin and synthesis of AuNPs. Reprinted from Mater Sc Eng C. Vol 90. Patra N, Dehury N, Pal A, Behera A, Patra S. Preparation and mechanistic aspect of natural xanthone functionalized gold nanoparticle, pages 439445, Copyright 2018, with permission from Elsevier. ${ }^{56}$ (C) Biosynthesis of AuNPs from Dragon fruit extract (DF extract) can be considered as an eco-friendly alternative for synthesis of AuNPs. Reprinted from Mater Lett. Vol 236. Divakaran D, Lakkakula JR, Thakur M, Kumawat MK, Srivastava R. Dragon fruit extract capped gold nanoparticles: synthesis and their differential cytotoxicity effect on breast cancer cells, pages 498-502, Copyright 2019, with permission from Elsevier. ${ }^{51}$ (D) One pot green synthesize of AuNPs were achieved using the leaf extracts of Carica papaya (CP) and Catharanthus roseus (CR) and the combination of these two extracts (CPCRM). Reprinted from Process Biochem. Vol 5I. Muthukumar T, Sudhakumari SB, Aravinthan A, Sastry TP, Kim JH. Green synthesis of gold nanoparticles and their enhanced synergistic antitumor activity using HepG2 and MCF7 cells and its antibacterial effects, pages 384-391, Copyright 2016, with permission from Elsevier. ${ }^{76}$ (E) Pectin, an anionic polysaccharide isolated from Musa paradisiaca is employed for the synthesis of AuNPs at ambient temperature conditions. Reprinted from Int J Biol Macromol. Vol 93. Suganya KSU, Govindaraju K, Kumar VG, Karthick V, Parthasarathy K. Pectin mediated gold nanoparticles induces apoptosisin mammary adenocarcinoma cell lines, pages 1030-1040, Copyright 2016, with permission from Elsevier. ${ }^{75}$ (F) Green synthesis of AuNPs from Anacardium occidental leaves extract. The colour change from gold to ruby red indicates the formation of AuNPs. Reprinted from Saudi J Biol Sci. Vol 26. Sunderam V, Thiyagarajan D, Lawrence AV, Mohammed SSS, Selvaraj A. In-vitro antimicrobial and anticancer properties ofgreen synthesized gold nanoparticles using Anacardium occidentale leaves extract, pages 455-459, Copyright 20I9, with permission from Elsevier. ${ }^{47}$

the significant anticancer potential of biogenic AuNPs against breast cancer cells through in vitro studies.

\section{Discussion}

\section{Cytotoxicity of Biogenic AuNPs Against Breast Cancer and Normal Cells}

The current study reports a systematic review of the efficacy of biogenic AuNPs, prepared using different natural sources (Figure 2) towards the spread of breast cancer. The findings showed a total of 4 trends with clear relevance in the study. The first group reported the cytotoxic effect of biogenic AuNPs towards breast cancer cells, while the second one reported no cytotoxicity at all when the cancerous tissue was exposed to the nanostructures. The third group presented significant cytotoxic effects of the biogenic AuNPs against breast cancer cells, but low or no cytotoxicity against healthy cells. The fourth group reported no cytotoxicity against both cancerous and standard cells. Importantly, no studies were found to report higher cytotoxicity of the biogenic AuNPs in healthy cells compared to breast cancer cells. As a consequence, this review shows a promising advance for the employment of biogenic AuNPs, which had less cytotoxicity when healthy cells were exposed compared to cancerous cells. 

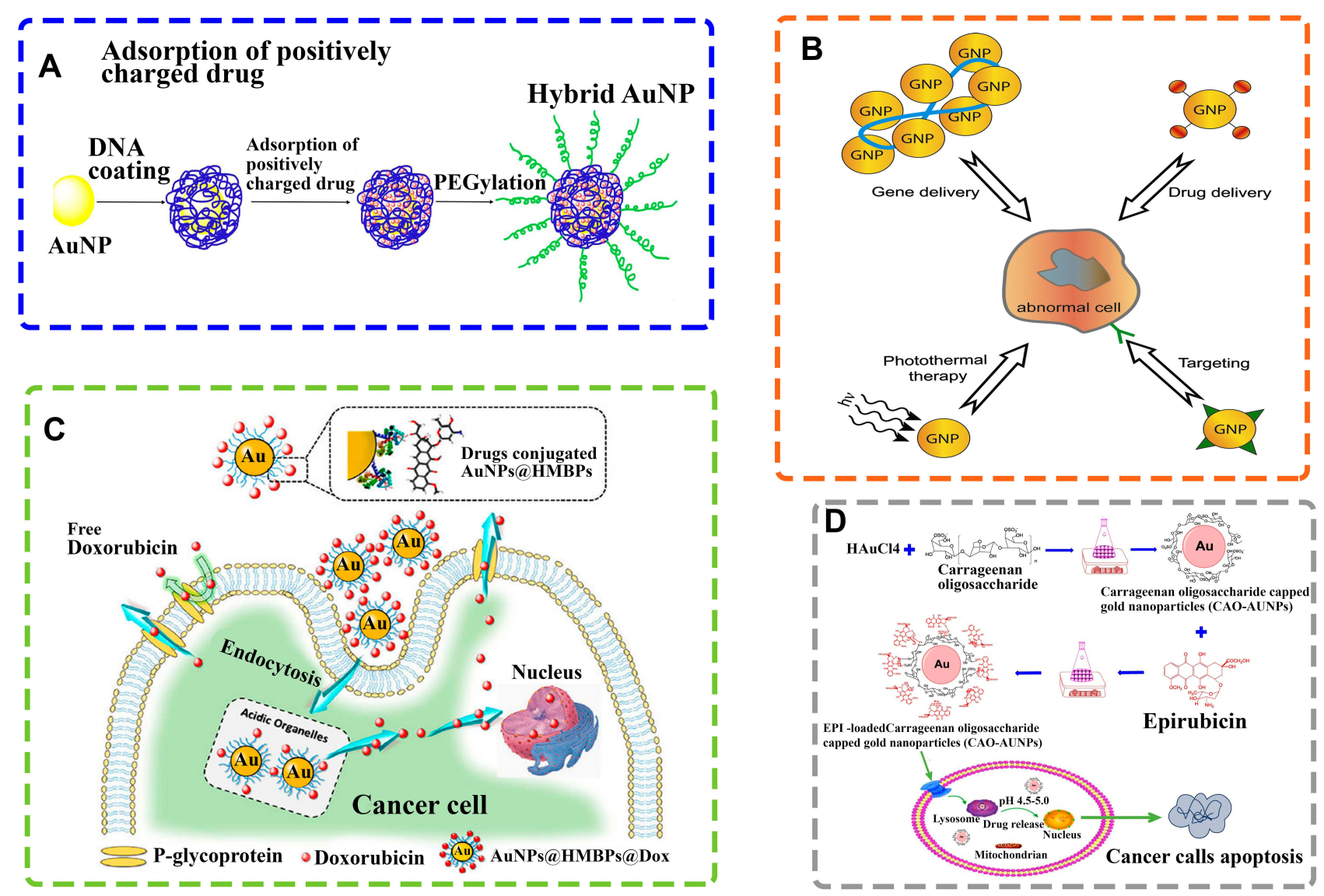

Figure 3 Schematic illustration of AuNPs for drug delivery systems. (A) Schematic illustration of hybrid AuNP coated by DNA, followed by the loading of positively charged drugs and then PEGylation for combination therapy of cancer. Reprinted from J Controlled Release. Vol 219. He C, Lu J, Lin W. Hybrid nanoparticles for combination therapy of cancer, pages 224-236, Copyright 2015, with permission from Elsevier. ${ }^{100}$ (B) Schematic illustration of different applications of AuNPs in diagnosis and therapy. AuNPs are used in a variety of contexts such as: photo thermal therapy, targeting, drug delivery, imaging, nucleic acid delivery, toxin and microbial agent removal and as an adjuvant. Reprinted from Adv Drug Delivery Rev. Vol 60. Ghosh P, Han G, De M, Kim CK, Rotello VM. Gold nanoparticles in delivery applications, pages I307-I5, Copyright 2008, with permission from Elsevier. ${ }^{101}$ (C) Schematic representation of synthesis of biogenic AuNPs and subsequent conjugation of doxorubicin. Biodegradable doxorubicin-loaded biogenic AuNPs complexes can be easily fragmented to release doxorubicin from AuNPs. Diffusion and accumulation of doxorubicin into cell nucleus could be achievable regardless of the size of AuNP used. Reprinted from Colloids Surf B Biointerfaces. Vol 135. Seo JM, Kim EB, Hyun MS, Kim BB, Park TJ. Self-assembly of biogenic gold nanoparticles and their use to enhance drug delivery into cells, pages 27-34, Copyright 2015, with permission from Elsevier. ${ }^{102}$ (D) Schematic diagram of epirubicin-loaded marine carrageenan oligosaccharide capped AuNPs for anticancer drug (epirubicin) delivery to combat cancer cells. Reprinted from Sci Rep. Vol 9. Chen X, Han W, Zhao X, Tang W, Wang F. Epirubicin-loaded marine carrageenan oligosaccharide capped gold nanoparticle system for $\mathrm{pH}$-triggered anticancer drug release, pages 6754, Copyright 2019, Under the terms of the Creative Commons CC BY license, Springer Nature. ${ }^{103}$

Furthermore, an important finding in terms of the raw material employed for the reduction and capping of the NPs was reported in this article. The overall studies showed that the different biological sources for the fabrication of AuNPs affect their cytotoxicity. For instance, Vijayakumar et al reported a plant-mediated synthesis of AuNPs using three different plants (Aegle marmelos, Eugenia jambolana, and Soursop). The different nanostructures showed variable cytotoxicity against MCF-7 cells, with $\mathrm{IC}_{50}$ values of $172 \pm 4,163 \pm 4$, and $98 \pm 4 \mu \mathrm{g} /$ $\mathrm{mL}$, respectively. ${ }^{53}$ Similarly, Muthukumar et al produced AuNPs using two different plants: Carica papaya (C. papaya) and Catharanthus roseus (C. roseus) and compared the cytotoxicity of the NPs towards MCF-7 and standard 3T3 cells using an MTT assay. After $24 \mathrm{~h}$ of incubation, the AuNPs synthesized from C. papaya showed an $\mathrm{IC}_{50}$ close to $100 \mu \mathrm{g} / \mathrm{mL}$ against MCF-7, while the ones produced from C. roseus showed an $\mathrm{IC}_{50}$ around $150 \mu \mathrm{g} / \mathrm{mL}$. Besides, the AuNPs synthesized from the combination of both plant extracts rendered $\mathrm{IC}_{50}$ values less 100 $\mu \mathrm{g} / \mathrm{mL}$ against MCF-7 cells. Surprisingly, when the NPs were produced by C. papaya, C. roseus, and a combination of these plants, they did not show any cytotoxicity towards 3T3 at concentrations of $250 \mu \mathrm{g} / \mathrm{mL}^{76}$

Remarkably, the studies also reported different cytotoxicity among different breast cancer cells. For example, Divakaran et al evaluated the cytotoxicity of plant-mediated synthesized AuNPs against MCF-7 and MDA-MB-231 breast cancer cells using an alamarBlue ${ }^{\circledR}$ assay. The results showed an $\mathrm{IC}_{50}$ value of around $500 \mu \mathrm{g} / \mathrm{mL}$, and less than 250 against MCF-7 cells 


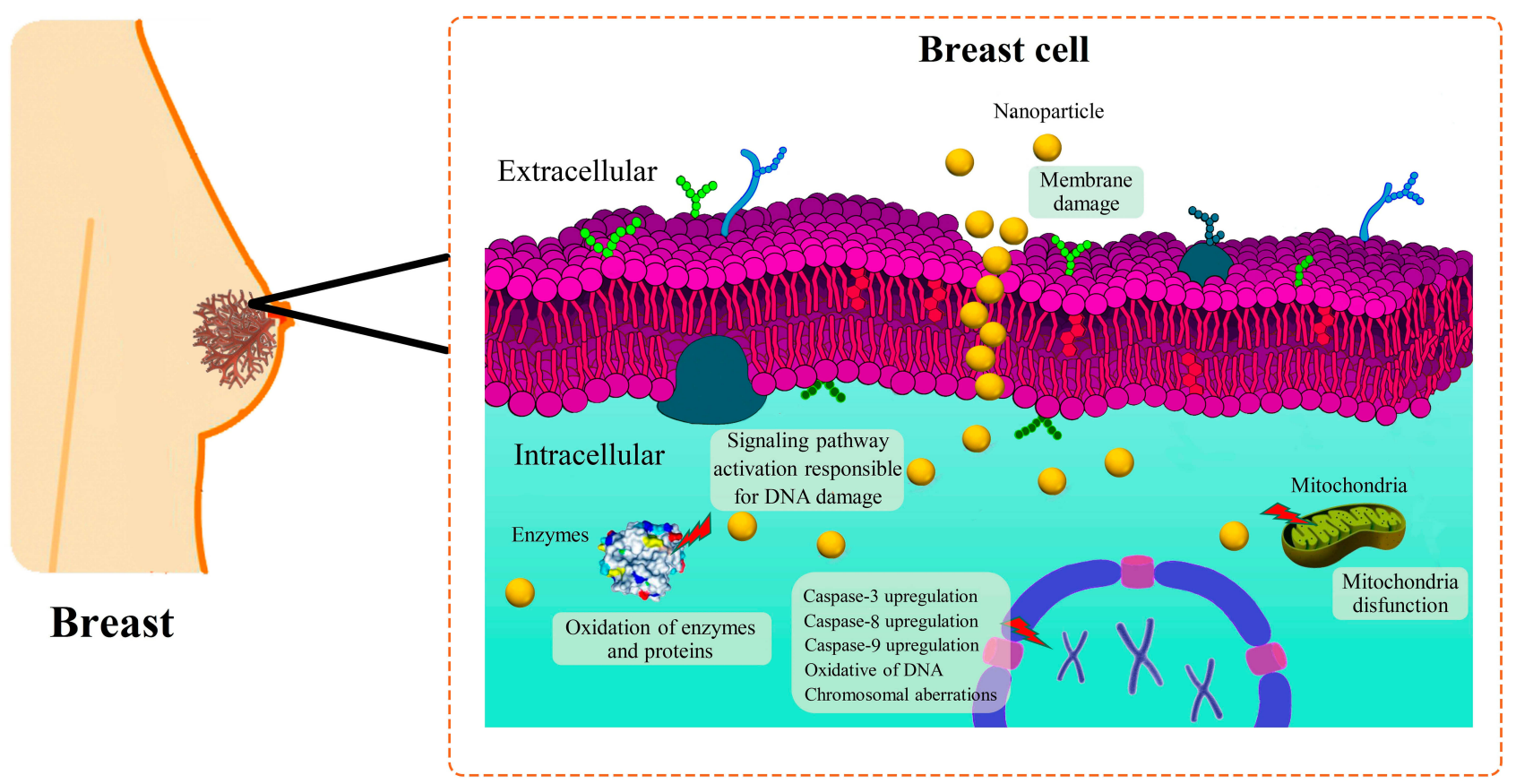

Figure 4 Schematic anti-cancer mechanism of AuNPs to combat breast cancer.

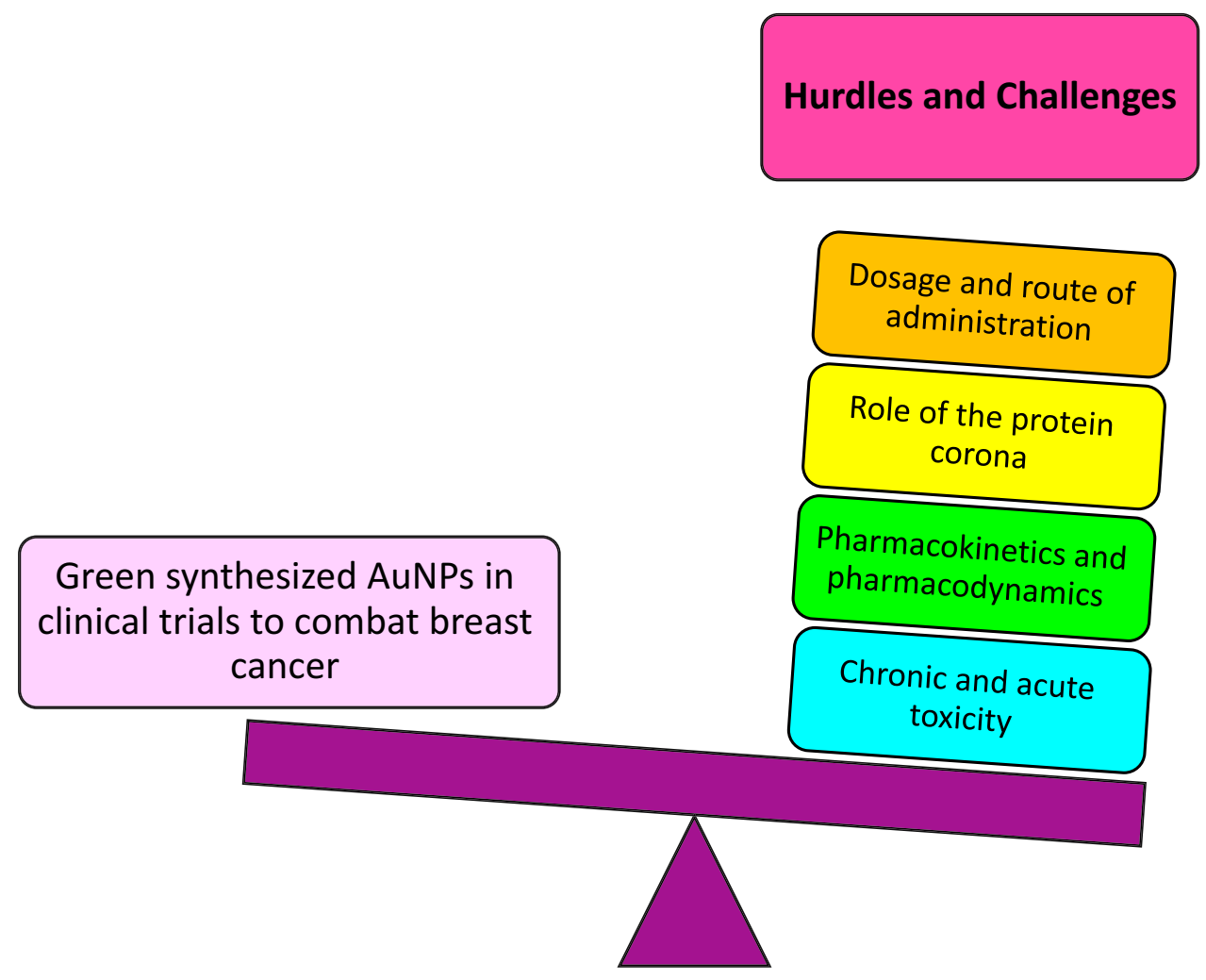

Figure 5 Hurdles and challenges of scientists before biogenic AuNPs enter clinical trials to combat breast cancer. 
after 24 and $48 \mathrm{~h}$ of incubation respectively, while no cytotoxicity was found against MDA-MB-231 cells at the same incubation periods. ${ }^{51}$ Similarly, Suganya et al compared the cytotoxicity of biogenic AuNPs against MCF-7 and MDAMB-231 breast cancer cells using an MTT assay. The authors reported an $\mathrm{IC}_{50}$ of around 8 and $2 \mu \mathrm{g} / \mathrm{mL}$ against MCF-7 and MDA-MB-231 after $48 \mathrm{~h}$ of incubation. However, no $\mathrm{IC}_{50}$ was reported after one day of incubation against both breast cancer cell lines. ${ }^{75}$ In a similar study, the $\mathrm{IC}_{50}$ value of plant-mediated AuNPs was found to be found 6 and $4 \mu \mathrm{g} / \mathrm{mL}$ after $48 \mathrm{~h}$ of incubation when the NPs were tested against MCF-7 and MDAMB-231 cancerous cells. However, no $\mathrm{IC}_{50}$ value was found after $24 \mathrm{~h}$ of incubation against both breast cancer cell lines. ${ }^{74}$

Interestingly, the AuNPs presented significant cytotoxicity against breast cancer cells, but less or no cytotoxicity towards normal cells. For instance, Sunderam et al investigated the cytotoxicity of plant-mediated AuNPs towards MCF-7 cells and peripheral blood mononuclear cells (PBMCs) using an MTT assay, reporting an $\mathrm{IC}_{50}$ of 6 and $600 \mu \mathrm{g} / \mathrm{mL}$, respectively after $24 \mathrm{~h}$ of incubation indicating a significant cytotoxicity of the NPs when tested towards MCF-7 cells compared to PBMCs. ${ }^{47}$ In a similar manner, Singh et al compared the cytotoxicity of algae-mediated AuNPs against cancerous MCF-7 and normal MCF-10A breast cells using the MTT assay after 24 and $48 \mathrm{~h}$ of incubation. The results of this study showed a clear time- and dosedependent cytotoxicity trend. After $24 \mathrm{~h}$ of incubation, around $65 \%$ of MCF-7 and MCF-10A cells were viable at the maximum concentration of study $(200 \mu \mathrm{g} / \mathrm{mL})$. After 48 $\mathrm{h}$ of incubation, around $55 \%$ of MCF-10A cells were viable at $200 \mu \mathrm{g} / \mathrm{mL}$, while about $15 \%$ of MCF-7 cells were viable at $200 \mu \mathrm{g} / \mathrm{mL}^{48}$ Besides, Safarpoor et al reported significant anticancer activity against MDA-MB-231 cells with an $\mathrm{IC}_{50}$ value of $1.67 \mu \mathrm{g} / \mathrm{mL}$, but lower cytotoxicity towards healthy cell lines, with an $\mathrm{IC}_{50}$ value of $4.85 \mu \mathrm{g} / \mathrm{mL}$ after $24 \mathrm{~h}$ of incubation. ${ }^{55}$ In a similar study, Jafari et al reported the bacteria-mediated synthesis of AuNPs and showed their cytotoxicity using an MTT assay against MCF-7, reporting an $\mathrm{IC}_{50}$ value of $105.3 \pm 1.7 \mu \mathrm{g} / \mathrm{mL}$ after $24 \mathrm{~h}$ of incubation, while no cytotoxicity was observed against $3 \mathrm{~T} 3$ cells at $500 \mu \mathrm{g} /$ $\mathrm{mL}$. $^{61}$ Besides, Barai et al investigated the cytotoxicity of plant-mediated AuNPs using the MTT assay. The authors reported an $\mathrm{IC}_{50}$ of $74.04 \mu \mathrm{g} / \mathrm{mL}$ against MCF-7 cells. At the same time, the NPs did not exhibit cytotoxicity against normal human lymphocytes at $200 \mu \mathrm{g} / \mathrm{mL} .^{66}$ Likewise, Balashanmugam et al reported a plant-mediated synthesis of AuNPs and evaluated their cytotoxicity against MCF-7 and healthy cells with an $\mathrm{IC}_{50}$ of around 50 and $75 \mu \mathrm{g} / \mathrm{mL}$, respectively, indicating much more cytotoxicity of AuNPs against MCF-7 cells. ${ }^{81}$ Furthermore, Bhat et al evaluated the cytotoxicity of fungi-mediated synthesized AuNPs towards MDA-MB and standard cells using an MTT assay. The results showed an $\mathrm{IC}_{50}$ of $39.1 \pm 3.17$ and $55.3 \pm 2.74 \mu \mathrm{g} / \mathrm{mL}$, respectively, after $72 \mathrm{~h}$ of incubation indicating far less cytotoxicity of AuNPs against healthy cells. ${ }^{94}$

A contrast was found within the studies that presented a lack of cytotoxic effects of biogenic AuNPs towards both healthy and cancerous cells. For example, Chokkalingam et al reported no cytotoxicity of plant-mediated AuNPs against cancerous MCF-7 and normal RAW264.7 cells at concentrations of 1 to $100 \mu \mathrm{g} / \mathrm{mL}$ after $48 \mathrm{~h}$ of incubation using the MTT assay. $^{52}$ These findings were in accordance to Soshnikova et $\mathrm{al}^{54}$ and Mukherjee et al, who evaluated the anticancer activity of plant-mediated AuNPs towards MCF-7 cells at concentrations of 2 and $20 \mu \mathrm{g} / \mathrm{mL}$ using an MTT assay. Furthermore, after $24 \mathrm{~h}$ of incubation, the NPs induced a 30$40 \%$ cytotoxicity at concentrations of 2 and $20 \mu \mathrm{g} / \mathrm{mL}$, respectively. ${ }^{84}$ Murugan et al evaluated the anticancer activity of bacterial-mediated AuNPs against MCF-7 cells and reported no cytotoxicity at a concentration of $200 \mathrm{mmol} / \mathrm{L}$ after $24 \mathrm{~h}$ of incubation. ${ }^{88}$ Moreover, Krishnaraj et al reported the anticancer activity of plans-mediated AuNPs against MDA-MB-231 cells using the MTT assay. The results showed that after $48 \mathrm{~h}$ of incubation at a concentration of $100 \mu \mathrm{g} / \mathrm{mL}$, the AuNPs induced $40 \%$ cytotoxicity against MDA-MB-231 cells. ${ }^{90}$ Alternatively, Fazal et al evaluated the cytotoxicity of photosynthesized AuNPs against cancerous MDA-MB-231 cells, and normal L929 and NIH-3T3 cells using an alamarBlue $^{\circledR}$ assay. The results showed no cytotoxicity of AuNPs after $24 \mathrm{~h}$ of incubation at $200 \mu \mathrm{g} / \mathrm{mL}$ against both cancer and normal cell lines. ${ }^{92}$ Similar studies were reported by Punuri et al and Babu et al, who evaluated the anticancer potential of photosynthesized AuNPs against MCF-7 using the MTT assay. According to these two studies, no cytotoxicity was found against MCF-7 cells in the range of 1 to $100 \mu \mathrm{mol} /$ L. ${ }^{95,97}$ Besides, Nune et al reported no cytotoxicity of plantmediated AuNPs against MCF-7 cells in the range of 10-150 $\mu \mathrm{mol} / \mathrm{L}$ after $24 \mathrm{~h}$ of incubation using the MTT assay. ${ }^{99}$

\section{Synergistic Effects of Biogenic AuNPs Conjugated to Anticancer Drugs to Combat Breast Cancer Cells}

The synergetic effects of biogenic AuNPs with therapeutic agents might be presented as a novel strategy in the future 
for targeted drug delivery systems, hence preventing unwanted dose-related side-effects (Figure 3).

Ranjith Santhosh Kumar et al synthesized AuNPs using the fungus Ganoderma lucidum in a straightforward and costeffective manner. Then, they compared the anticancer activity of doxorubicin (DOX) with the biosynthesized NPs conjugated to DOX using the MTT assay. The DOX concentration range was set between 6.25 and $300 \mu \mathrm{mol} / \mathrm{L}$, with measurements taken after $48 \mathrm{~h}$ of incubation. The $\mathrm{IC}_{50}$ value of the AuNPs conjugated to the chemotherapy drug was $50 \mu \mathrm{M}$, while the $\mathrm{IC}_{50}$ value of DOX alone was $400 \mu \mathrm{mol} / \mathrm{L}$, which clearly indicates the influence and relevance of the combination of biogenic AuNPs with anticancer drugs, reducing cytotoxicity. ${ }^{69}$ Alternatively, Kumar et al followed a green-synthesis approach for the production of AuNPs using the plant Limonia acidissima $\mathrm{L}$. and conjugated them to epirubicin and folic acid (FA). They then compared the anticancer activity of these complexes with free epirubicin, testing the system towards MCF-7 cells, with a drug range up to $30 \mu \mathrm{g} / \mathrm{mL}$ for 2 days of incubation. The $\mathrm{IC}_{50}$ value of this complex was $2 \mu \mathrm{g} / \mathrm{mL}$, while the $\mathrm{IC}_{50}$ value of the free epirubicin was $28 \mu \mathrm{g} / \mathrm{mL}{ }^{78}$ In a different way, Ganeshkumar et al produced AuNPs using the plant Punica granutum and conjugated the nanostructures to 5-fluorouracil (5-Fu) and FA for the evaluation of their anticancer activity against MCF-7 cells using the MTT assay. The $\mathrm{IC}_{50}$ value of conjugated the AuNPs was found at $250 \mathrm{ng} / \mathrm{mL}$ (concentration of 5-Fu), while the $\mathrm{IC}_{50}$ value of $5-\mathrm{Fu}$ was found to be $1000 \mathrm{ng} / \mathrm{mL}^{93}$ Beyond these findings, further studies are required to evaluate the anticancer potential of the biogenic AuNPs and some FDA approved drugs through in vivo models.

\section{Proposed Molecular Mechanisms of Biogenic AuNPs-Induced Cytotoxicity Against Breast Cancer Cells}

Although the exact molecular mechanisms of biogenic AuNPs-induced cytotoxicity against breast cancer cells is not fully understood, some studies proposed the involvement of different molecular mechanisms, such as the influence of Reactive Oxygen Species (ROS) and apoptosis factors as shown in Figure 4.

\section{ROS-Induced Cytotoxicity Mechanism}

Reactive oxygen species, or ROS, are unstable free radicals that contain oxygen and easily react with other molecules presented in cellular environments, leading to different alterations, such as cell damage at the molecular level, which may lead to cell death. Some researchers have found that there exists a clear biogenic AuNPs-induced overproduction of intracellular ROS when they contact biological tissue. ${ }^{66}$ A study was done by Barai et al, where they measured intracellular ROS levels after exposure of biogenic AuNPs to breast cancer cells, showing that the plant-mediated NPs caused significant cytotoxicity towards MCF-7 cells at a concentration of $100 \mu \mathrm{g} / \mathrm{mL}$ due to the overproduction of ROS. ${ }^{66}$ Likewise, Parveen et al confirmed that biogenic AuNPs induced cytotoxicity against MDA-MB cells due to an excess of ROS levels. The authors also reported that ROS might result in deoxyribose phosphate backbone damage as well as a change in purine and pyrimidine bases through the chemical modifications in DNA, ${ }^{83}$ which undoubtedly led to cell death.

\section{AIF-Induced Cytotoxicity Mechanism}

Apoptosis-inducing factors (AIFs) are proteins which trigger chromatin condensation and DNA fragmentation in a particular cell, leading to programmed cell death. The production of these factors was found to be related to exposure of the cells to external agents, such as NPs. A recent study reported the production of algae-mediated AuNPs and the induction of late apoptosis when they were exposed to MCF7 cancer cells after a $24 \mathrm{~h}$ incubation period. ${ }^{48}$ In a similar study, a meticulous cell cycle analysis revealed significant apoptosis in the G0/G1 to S cell phases when MDA-MB-231 and MCF-7 cells were exposed to plant-mediated AuNPs. These apoptotic cycles led to DNA damage inside the cancerous cells, reporting $\mathrm{IC}_{50}$ values $(6 \mu \mathrm{g} / \mathrm{mL}$ for MCF-7 and $4 \mu \mathrm{g} /$ $\mathrm{mL}$ for MDA-MB-231 cells) after $48 \mathrm{~h}$ of incubation. The study also revealed considerable numbers of nucleoids with massive tails, indicating a high level of DNA single-strand breaks within the cells. ${ }^{74}$ Besides, it was reported that MCF7 cells treated with photosynthesized AuNPs showed a considerable decline in the intracellular glutathione content and an increase in the $\mathrm{Bax} / \mathrm{Bcl} 2$ ratio and caspase-3 (proteins related to apoptosis). ${ }^{84}$ In a similar study, the plant-mediated AuNPs showed a dose-dependent increase in caspase-3, -8 , and -9 protease activities in MCF-7 cells which were treated with different concentrations of AuNPs, ranging from 6.25 to $100 \mu \mathrm{g} / \mathrm{mL}$ for $48 \mathrm{~h} .{ }^{87}$ Likewise, a different study reported increased levels of caspase- 3 protease activation in MDA-MB -231 cells treated with plant-synthesized AuNPs. ${ }^{90}$

\section{Biogenic AuNPs as Future Anti-Cancer Nanomedicine: Hurdles and Challenges}

Figure 5 shows the challenges of AuNPs to enter clinical trials in the fight towards breast cancer. Although different NPs are 
presented as a promising strategy for the next-generation of theranostic drugs, their safety remains a concern for human patients, with no exception for biogenic AuNPs. As it was stated before, NMs' properties are different from their bulk counterparts. ${ }^{41}$ The size, shape, surface charge, surface chemistry, and other properties of biogenic NPs may change their pharmacokinetics and pharmacodynamics as well as their cytotoxicity and presence of side-effects. ${ }^{12-14}$ Nonetheless, it is well-known that green synthesized NMs present advances in terms of biocompatibility and generation, leading to a new variety of possibilities and enhancements. Despite that, many challenges should be addressed before the translation of laboratory studies to clinical applications. The chronic and acute toxicity of biogenic AuNPs should be considered in future studies.

Moreover, a comprehensive study should be carried out concerning the role of the protein corona surrounding the spheres. The protein corona is made of biomolecules from the natural raw materials used for the production of the NPs, such as lipids, proteins, carbohydrates and other small molecules. ${ }^{104,105}$ Therefore, the protein corona has a strong influence on the surface chemistry and charge, clearly leading to different responses in cellular uptake, cytotoxicity, immunogenicity, and anticancer activity of the biogenic AuNPs. ${ }^{106,107}$ Besides, the green NPs can be conjugated to other FDA approved drugs, as well as different therapeutic and diagnostic agents, enhancing their biomedical applications. Besides, research has shown the promising future of biogenic AuNPs as drug delivery nanocarriers, ${ }^{13}$ which undoubtedly provides a path for designing the next-generation of nanoplatforms for medical applications. Furthermore, the role of natural-derived reducing and stabilizing agents on the cytotoxicity of biogenic AuNPs should be explained in future studies. Additionally, the mammalian cell based capacities to synthesize AuNPs were recently investigated as a promising strategy for bioimaging, hyperthermia and other therapeutic applications. ${ }^{108-110}$ Despite all the mentioned challenges, it is expected that more and more biogenic AuNPs will be employed as anticancer drugs, either alone as carriers or combined with drugs.

\section{Conclusion}

The current review reported a systematic effort to discuss the efficacy of biologically synthesized AuNPs against breast cancer cells through in vitro investigations. The studies presented several naturally-derived raw materials used as unique reducing and capping agents for the environmentally-friendly and cost-effective synthesis of AuNPs with different morphologies and size distributions. Although many heterogeneities have been reported throughout these studies, an inclusive approach presented much more cytotoxicity of biogenic AuNPs towards breast cancer cells compared to healthy cells. Besides, the biogenic AuNPs showed significant synergy when combined with other anticancer drugs through in vitro models. The proposed anticancer mechanisms of biogenic AuNPs included the overproduction of ROS, as well as activation of caspase cascades for induction of apoptosis. Although preliminary evidence of the significance of the anticancer potential of ecofriendly synthesized AuNPs was presented, further in vivo studies should be conducted to show their anticancer efficacy through animal models. Moreover, many challenges and ambiguities about biogenic AuNPs should be addressed in the future such as their genotoxicity, safety profile, therapeutic window, pharmacokinetics, and pharmacodynamics, among others.

\section{Acknowledgments}

This work was financially supported by Shahid Beheshti University of Medical Sciences, Tehran, Iran [Grant Number 19812].

\section{Disclosure}

The authors report no conflicts of interest in this work.

\section{References}

1. Siegel RL, Miller KD, Jemal A. Cancer statistics, 2019. CA Cancer J Clin. 2019;69(1):7-34. doi:10.3322/caac.21551

2. Zavala VA, Serrano-Gomez SJ, Dutil J, Fejerman L. Genetic epidemiology of breast cancer in Latin America. Genes (Basel). 2019;10 (2):153. doi:10.3390/genes 10020153

3. Benson JR, Jatoi I. The global breast cancer burden. Future Oncol. 2012;8(6):697-702. doi:10.2217/fon.12.61

4. Wu D, Si M, Xue HY, Wong HL. Nanomedicine applications in the treatment of breast cancer: current state of the art. Int J Nanomedicine. 2017;12:5879-5892. doi:10.2147/IJN.S123437

5. Bulut N. Histopathological characteristics: clinical course of breast cancer subtypes depending on the ER $(+)(-) / P R(+)(-)$ Receptor Status. In: Breast Cancer: From Biology to Medicine. IntechOpen. 2017;29.

6. Lu RM, Chen MS, Chang DK, et al. Targeted drug delivery systems mediated by a novel Peptide in breast cancer therapy and imaging. PLoS One. 2013;8(6):e66128. doi:10.1371/journal.pone.0066128

7. Heldin $\mathrm{CH}$, Rubin K, Pietras K, Ostman A. High interstitial fluid pressure - an obstacle in cancer therapy. Nat Rev Cancer. 2004;4 (10):806-813. doi:10.1038/nrc1456

8. Rebucci M, Michiels C. Molecular aspects of cancer cell resistance to chemotherapy. Biochem Pharmacol. 2013;85(9):1219-1226. doi:10.1016/j.bcp.2013.02.017

9. Gottesman MM, Pastan IH. The role of multidrug resistance efflux pumps in cancer: revisiting a JNCI publication exploring expression of the MDR1 (P-glycoprotein) gene. J Natl Cancer Inst. 2015;107(9): djv222. doi:10.1093/jnci/djv222 
10. Xue X, Liang X-J. Overcoming drug efflux-based multidrug resistance in cancer with nanotechnology. Chin $J$ Cancer. 2012;31(2):100-109. doi:10.5732/cjc.011.10326

11. Tredan O, Galmarini CM, Patel K, Tannock IF. Drug resistance and the solid tumor microenvironment. J Natl Cancer Inst. 2007;99(19):1441-1454. doi:10.1093/jnci/djm135

12. Barabadi H, Mahjoub MA, Tajani B, Ahmadi A, Junejo Y, Saravanan M. Emerging theranostic biogenic silver nanomaterials for breast cancer: a systematic review. J Cluster Sci. 2019;30 (2):259-279. doi:10.1007/s10876-018-01491-7

13. Barabadi H, Damavandi Kamali K, Jazayeri Shoushtari F, et al. Emerging theranostic silver and gold nanomaterials to combat prostate cancer: a systematic review. J Cluster Sci. 2019.

14. Barabadi H, Hosseini O, Damavandi Kamali K, et al. Emerging theranostic silver nanomaterials to combat lung cancer: a systematic review. J Cluster Sci. 2019.

15. Mostafavi E, Soltantabar P, Webster TJ. Nanotechnology and picotechnology: a new arena for translational medicine. Biomater Transl Med. 2019;191-212.

16. Grobmyer SR, Iwakuma N, Sharma P, Moudgil BM. What is cancer nanotechnology? Meth Mol Biol. 2010;624:1-9.

17. Rocha M, Chaves N, Báo S. Nanobiotechnology for Breast Cancer Treatment. In: Breast Cancer-From Biology to Medicine. IntechOpen; 2017. Available from: https://www.intechopen.com/ books/breast-cancer-from-biology-to-medicine/nanobiotechnol ogy-for-breast-cancer-treatment. Accessed May 15, 2019.

18. Yonezawa T. Application 78 - preparation of metal nanoparticles and their application for materials. In: Naito M, Yokoyama T, Hosokawa K, Nogi K, editors. Nanoparticle Technology Handbook. 3rd ed. Elsevier; 2018:829-837.

19. Wenjie M, Qiong W. Applications of metal nanoparticles in medicine/metal nanoparticles as anticancer agents. In: Metal Nanoparticles Germany. Wiley-VCH. 2017;169-190.

20. Barabadi H, Honary S, Ebrahimi P, Alizadeh A, Naghibi F, Saravanan M. Optimization of myco-synthesized silver nanoparticles by response surface methodology employing Box-Behnken design. Inorganic Nano Metal Chem. 2019;49(2):33-43. doi:10.1080/24701556.2019.1583251

21. Salari S, Esmaeilzadeh Bahabadi S, Samzadeh-Kermani A, Yousefzaei F. In vitro evaluation of antioxidant and antibacterial potential of green synthesized silver nanoparticles using Prosopis farcta fruit extract. Iran J Pharm Res. 2019;18(1):430-445.

22. Rezvani Amin Z, Khashyarmanesh Z, Fazly Bazzaz BS, Sabeti Noghabi Z. Does biosynthetic silver nanoparticles are more stable with lower toxicity than their synthetic counterparts? Iran J Pharm Res. 2019;18(1):210-221.

23. Ramezani T, Nabiuni M, Baharara J, Parivar K, Namvar F. Sensitization of resistance ovarian cancer cells to cisplatin by biogenic synthesized silver nanoparticles through p53 activation. Iran J Pharm Res. 2019;18(1):222-231.

24. Miri B, Motakef-Kazemi N, Shojaosadati SA, Morsali A. Application of a nanoporous metal organic framework based on iron carboxylate as drug delivery system. Iran J Pharm Res. 2018;17(4):1164-1171.

25. Barabadi H, Kobarfard F, Vahidi H. Biosynthesis and characterization of biogenic tellurium nanoparticles by using Penicillium chrysogenum PTCC 5031: a novel approach in gold biotechnology. Iran J Pharm Res. 2018;17:87-97.

26. maham M, Karami-Osboo R. Extraction of sulfathiazole from urine using biosynthesized magnetic nanoparticles. Iran J Pharm Res. 2017;16(2):462-470.

27. Karimi N, Chardoli A, Fattahi A. Biosynthesis, characterization, antimicrobial and cytotoxic effects of silver nanoparticles using nigella arvensis seed extract. Iran J Pharm Res. 2017;16(3):1167-1175.

28. Dobrucka R. Synthesis of titanium dioxide nanoparticles using Echinacea purpurea herba. Iran J Pharm Res. 2017;16(2):753-759.
29. Abbas Q, Saleem M, Phull AR, et al. Green synthesis of silver nanoparticles using bidens frondosa extract and their tyrosinase activity. Iran J Pharm Res. 2017;16(2):760-767.

30. Or Rashid MM, Islam MS, Haque MA, Rahman MA, Hossain MT, Hamid MA. Antibacterial activity of polyaniline coated silver nanoparticles synthesized from piper betle leaves extract. Iran J Pharm Res. 2016;15(2):591-597.

31. Vernet Crua A, Medina D, Zhang B, et al. Comparison of cytocompatibility and anticancer properties of traditional and green chemistry-synthesized tellurium nanowires. Int J Nanomedicine. 2019;14:3155-3176. doi:10.2147/IJN.S175640

32. Medina Cruz D, Tien-Street W, Zhang B, et al. Citric juice-mediated synthesis of tellurium nanoparticles with antimicrobial and anticancer properties. Green Chem. 2019;21 (8):1982-1998. doi:10.1039/C9GC00131J

33. Lomelí-Marroquín D, Medina Cruz D, Nieto-Argüello A, et al. Starchmediated synthesis of mono- and bimetallic silver/gold nanoparticles as antimicrobial and anticancer agents. Int $J$ Nanomedicine. 2019;14:2171-2190. doi:10.2147/IJN.S192757

34. Medina Cruz D, Mi G, Webster TJ. Synthesis and characterization of biogenic selenium nanoparticles with antimicrobial properties made by Staphylococcus aureus, methicillin-resistant Staphylococcus aureus (MRSA), Escherichia coli, and Pseudomonas aeruginosa. J Biomed Mater Res A. 2018;106(5):1400-1412. doi:10.1002/jbm. a.36347

35. Yadi M, Mostafavi E, Saleh B, et al. Current developments in green synthesis of metallic nanoparticles using plant extracts: a review. Artif Cells Nanomedicine Biotechnol. 2018;46(sup3): S336-S343. doi:10.1080/21691401.2018.1492931

36. Kasithevar M, Saravanan M, Prakash P, et al. Green synthesis of silver nanoparticles using Alysicarpus monilifer leaf extract and its antibacterial activity against MRSA and CoNS isolates in HIV patients. J Interdiscipl Nanomedicine. 2017;2(2):131-141. doi:10.1002/jin2.26

37. Barabadi H, Honary S. Biofabrication of gold and silver nanoparticles for pharmaceutical applications. Pharm Biomed Res. 2016;2(1):1-7. doi:10.18869/acadpub.pbr.2.1.1

38. Amani H, Mostafavi E, Alebouyeh MR, et al. Would colloidal gold nanocarriers present an effective diagnosis or treatment for ischemic stroke? Int J Nanomedicine. 2019;14:8013. doi:10.2147/ IJN.S210035

39. Hornos Carneiro MF, Barbosa F Jr. Gold nanoparticles: a critical review of therapeutic applications and toxicological aspects. $J$ Toxicol Environ Health B Crit Rev. 2016;19(3-4):129-148. doi:10.1080/10937404.2016.1168762

40. Ghosh P, Han G, De M, Kim CK, Rotello VM. Gold nanoparticles in delivery applications. Adv Drug Deliv Rev. 2008;60 (11):1307-1315. doi:10.1016/j.addr.2008.03.016

41. Saravanan M, Asmalash T, Gebrekidan A, et al. Nano-medicine as a newly emerging approach to combat Human Immunodeficiency Virus (HIV). Pharm Nanotechnol. 2018;6(1):17-27. doi:10.2174/ 2211738506666180209095710

42. Hainfeld JF, Dilmanian FA, Zhong Z, Slatkin DN, Kalef-Ezra JA, Smilowitz HM. Gold nanoparticles enhance the radiation therapy of a murine squamous cell carcinoma. Phys Med Biol. 2010;55 (11):3045-3059. doi:10.1088/0031-9155/55/11/004

43. Hainfeld JF, Slatkin DN, Smilowitz HM. The use of gold nanoparticles to enhance radiotherapy in mice. Phys Med Biol. 2004;49(18):N309-315. doi:10.1088/0031-9155/49/18/N03

44. Qian X, Peng X-H, Ansari DO, et al. In vivo tumor targeting and spectroscopic detection with surface-enhanced Raman nanoparticle tags. Nat Biotechnol. 2007;26:83. doi:10.1038/nbt1377

45. Eck W, Nicholson AI, Zentgraf H, Semmler W, Bartling S. Anti-CD4targeted gold nanoparticles induce specific contrast enhancement of peripheral lymph nodes in X-ray computed tomography of live mice. Nano Lett. 2010;10(7):2318-2322. doi:10.1021/nl101019s 
46. Moher D, Liberati A, Tetzlaff J, Altman DG. Preferred reporting items for systematic reviews and meta-analyses: the PRISMA statement. PLoS Med. 2009;6(7):e1000097. doi:10.1371/journal. pmed. 1000097

47. Sunderam V, Thiyagarajan D, Lawrence AV, Mohammed SSS, Selvaraj A. In-vitro antimicrobial and anticancer properties of green synthesized gold nanoparticles using Anacardium occidentale leaves extract. Saudi J Biol Sci. 2019;26(3):455-459. doi:10.1016/j.sjbs.2018.12.001

48. Singh AK, Tiwari R, Singh VK, et al. Green synthesis of gold nanoparticles from Dunaliella salina, its characterization and in vitro anticancer activity on breast cancer cell line. J Drug Deliv Sci Technol. 2019;51:164-176. doi:10.1016/j.jddst.2019.02.023

49. Majumdar M, Biswas SC, Choudhury R, et al. Synthesis of gold nanoparticles using citrus macroptera fruit extract: anti-biofilm and anticancer activity. ChemistrySelect. 2019;4(19):5714-5723. doi:10.1002/slct.201804021

50. Hamed MM, Abdelftah LS. Biosynthesis of gold nanoparticles using marine streptomyces griseus isolate (M8) and evaluating its antimicrobial and anticancer activity. Egypt $J$ Aqua Biol Fisheries. 2019;23(1):173-184. doi:10.21608/ejabf.2019.26508

51. Divakaran D, Lakkakula JR, Thakur M, Kumawat MK, Srivastava R. Dragon fruit extract capped gold nanoparticles: synthesis and their differential cytotoxicity effect on breast cancer cells. Mater Lett. 2019;236:498-502. doi:10.1016/j.matlet.2018.10.156

52. Chokkalingam M, Singh P, Huo Y, et al. Facile synthesis of Au and Ag nanoparticles using fruit extract of Lycium chinense and their anticancer activity. J Drug Deliv Sci Technol. 2019;49:308-315. doi:10.1016/j.jddst.2018.11.025

53. Vijayakumar S. Eco-friendly synthesis of gold nanoparticles using fruit extracts and in vitro anticancer studies. J Saudi Chem Soc. 2019;23(6):753-761. doi:10.1016/j.jscs.2018.12.002

54. Soshnikova V, Kim YJ, Singh P, et al. Cardamom fruits as a green resource for facile synthesis of gold and silver nanoparticles and their biological applications. Artif Cells Nanomedicine Biotechnol. 2018;46 (1):108-117. doi:10.1080/21691401.2017.1296849

55. Safarpoor M, Ghaedi M, Yousefinejad M, et al. Podophyllotoxin extraction from Linum usitatissimum plant and its anticancer activity against HT-29, A-549 and MDA-MB-231 cell lines with and without the presence of gold nanoparticles. Appl Organomet Chem. 2018;32:2. doi:10.1002/aoc.4024

56. Patra N, Dehury N, Pal A, Behera A, Patra S. Preparation and mechanistic aspect of natural xanthone functionalized gold nanoparticle. Mater Sc Eng C. 2018;90:439-445. doi:10.1016/j. msec.2018.04.091

57. Oueslati MH, Tahar LB, Harrath AH. Catalytic, antioxidant and anticancer activities of gold nanoparticles synthesized by kaempferol glucoside from Lotus leguminosae. Arab J Chem. 2018.

58. Oh KH, Soshnikova V, Markus J, et al. Biosynthesized gold and silver nanoparticles by aqueous fruit extract of Chaenomeles sinensis and screening of their biomedical activities. Artif Cells Nanomedicine Biotechnol. 2018;46(3):599-606. doi:10.1080/ 21691401.2017.1332636

59. Li J, Tian B, Li T, et al. Biosynthesis of Au, Ag and Au-Ag bimetallic nanoparticles using protein extracts of Deinococcus radiodurans and evaluation of their cytotoxicity. Int $J$ Nanomedicine. 2018;13:1411-1424. doi:10.2147/IJN.S149079

60. Khandanlou R, Murthy V, Saranath D, Damani H. Synthesis and characterization of gold-conjugated Backhousia citriodora nanoparticles and their anticancer activity against MCF-7 breast and HepG2 liver cancer cell lines. J Mater Sci. 2018;53 (5):3106-3118. doi:10.1007/s10853-017-1756-4

61. Jafari M, Rokhbakhsh-Zamin F, Shakibaie M, et al. Cytotoxic and antibacterial activities of biologically synthesized gold nanoparticles assisted by Micrococcus yunnanensis strain J2. Biocatal Agri Biotechnol. 2018;15:245-253. doi:10.1016/j.bcab.2018.06.014
62. Ismail EH, Saqer AMA, Assirey E, Naqvi A, Okasha RM. Successful green synthesis of gold nanoparticles using a corchorus olitorius extract and their antiproliferative effect in cancer cells. Int J Mol Sci. 2018;19:9. doi:10.3390/ijms 19092612

63. Huo Y, Singh P, Kim YJ, et al. Biological synthesis of gold and silver chloride nanoparticles by Glycyrrhiza uralensis and in vitro applications. Artif Cells Nanomedicine Biotechnol. 2018;46 (2):303-312. doi:10.1080/21691401.2017.1307213

64. Chen X, Zhao X, Gao Y, Yin J, Bai M, Wang F. Green synthesis of gold nanoparticles using carrageenan oligosaccharide and their in vitro antitumor activity. Mar Drugs. 2018;16:8. doi:10.3390/md16080277

65. Chahardoli A, Karimi N, Sadeghi F, Fattahi A. Green approach for synthesis of gold nanoparticles from Nigella arvensis leaf extract and evaluation of their antibacterial, antioxidant, cytotoxicity and catalytic activities. Artif Cells Nanomedicine Biotechnol. 2018;46(3):579-588. doi:10.1080/21691401.2017.1332634

66. Barai AC, Paul K, Dey A, et al. Green synthesis of Nerium oleander-conjugated gold nanoparticles and study of its in vitro anticancer activity on MCF-7 cell lines and catalytic activity. Nano Convergence. 2018;5(1):10. doi:10.1186/s40580-018-0142-5

67. Ahn EY, Lee YJ, Choi SY, Im AR, Kim YS, Park Y. Highly stable gold nanoparticles green-synthesized by upcycling cartilage waste extract from yellow-nose skate (Dipturus chilensis) and evaluation of its cytotoxicity, haemocompatibility and antioxidant activity. Artif Cells Nanomedicine Biotechnol. 2018;46 (sup2):1108-1119. doi:10.1080/21691401.2018.1479710

68. Wang D, Markus J, Wang C, et al. Green synthesis of gold and silver nanoparticles using aqueous extract of Cibotium barometz root. Artif Cells Nanomedicine Biotechnol. 2017;45(8):1548-1555. doi:10.1080/ 21691401.2016.1260580

69. Ranjith Santhosh Kumar DS, Senthilkumar P, Surendran L, Sudhagar B. Ganoderma lucidum-oriental mushroom mediated synthesis of gold nanoparticles conjugated with doxorubicin and evaluation of its anticancer potential on human breast cancer mcf-7/dox cells. Int J Pharm Pharm Sci. 2017;9(9):267-274. doi:10.22159/ijpps.2017v9i9.20093

70. Firdhouse MJ, Lalitha P. Cytotoxicity of spherical gold nanoparticles synthesised using aqueous extracts of aerial roots of Rhaphidophora aurea (Linden ex Andre) intertwined over Lawsonia inermis and Areca catechu on MCF-7 cell line. IET Nanobiotechnol. 2017;11(1):2-11. doi:10.1049/iet-nbt.2016.0076

71. Dhayalan M, Denison MI, Krishnan LAJ, NG N. In vitro antioxidant, antimicrobial, cytotoxic potential of gold and silver nanoparticles prepared using Embelia ribes. Nat Prod Res. 2017;31(4):465-468. doi:10.1080/14786419.2016.1166499

72. Devi GK, Sathishkumar K. Synthesis of gold and silver nanoparticles using Mukia maderaspatna plant extract and its anticancer activity. IET Nanobiotechnol. 2017;11(2):143-151. doi:10.1049/iet-nbt.2015.0054

73. Ahmad N, Bhatnagar S, Saxena R, Iqbal D, Ghosh AK, Dutta R. Biosynthesis and characterization of gold nanoparticles: kinetics, in vitro and in vivo study. Mater Sc Eng C. 2017;78:553-564. doi:10.1016/j.msec.2017.03.282

74. Suganya KSU, Govindaraju K, Kumar VG, et al. Antiproliferative effect of biogenic gold nanoparticles against breast cancer cell lines (MDA-MB-231 \& MCF-7). Appl Surf Sci. 2016;371:415-424. doi:10.1016/j.apsusc.2016.03.004

75. Suganya KSU, Govindaraju K, Kumar VG, Karthick V, Parthasarathy K. Pectin mediated gold nanoparticles induces apoptosis in mammary adenocarcinoma cell lines. Int $J$ Biol Macromol. 2016;93:1030-1040. doi:10.1016/j.ijbiomac.2016.08.086

76. Muthukumar T, Sudhakumari SB, Aravinthan A, Sastry TP, Kim JH. Green synthesis of gold nanoparticles and their enhanced synergistic antitumor activity using HepG2 and MCF7 cells and its antibacterial effects. Process Biochem. 2016;51(3):384-391. doi:10.1016/j.procbio.2015.12.017 
77. Mmola M, Roes-Hill ML, Durrell K, et al. Enhanced antimicrobial and anticancer activity of silver and gold nanoparticles synthesised using sargassum incisifolium aqueous extracts. Molecules. 2016;21(12):1633. doi:10.3390/molecules21121633

78. Kumar C, Mahesh A, Antoniraj M, Vaidevi S, Ruckmani K. Ultrafast synthesis of stabilized gold nanoparticles using aqueous fruit extract of Limonia acidissima L. and conjugated epirubicin: targeted drug delivery for treatment of breast cancer. RSC Adv. 2016;6(32):26874-26882. doi:10.1039/C6RA01482H

79. Khan AU, Yuan Q, Wei Y, et al. Longan fruit juice mediated synthesis of uniformly dispersed spherical AuNPs: cytotoxicity against human breast cancer cell line MCF-7, antioxidant and fluorescent properties. RSC Adv. 2016;6(28):23775-23782. doi:10.1039/C5RA27100B

80. Kajani AA, Bordbar AK, Zarkesh Esfahani SH, Razmjou A. Gold nanoparticles as potent anticancer agent: green synthesis, characterization, and: in vitro study. RSC Adv. 2016;6(68):63973-63983. doi:10.1039/C6RA09050H

81. Balashanmugam P, Durai P, Balakumaran MD, Kalaichelvan PT. Phytosynthesized gold nanoparticles from C. roxburghii DC. leaf and their toxic effects on normal and cancer cell lines. J Photochem Photobiol B. 2016;165:163-173. doi:10.1016/j.jphotobiol.2016.10.013

82. Yallappa S, Manjanna J, Dhananjaya BL, Vishwanatha U, Ravishankar B, Gururaj H. Phytosynthesis of gold nanoparticles using Mappia foetida leaves extract and their conjugation with folic acid for delivery of doxorubicin to cancer cells. J Mater Sci Mater Med. 2015;26:9.

83. Parveen A, Cytotoxicity RS. Genotoxicity of biosynthesized gold and silver nanoparticles on human cancer cell lines. J Cluster Sci. 2015;26(3):775-788. doi:10.1007/s10876-014-0744-y

84. Mukherjee S, Ghosh S, Das DK, et al. Gold-conjugated green tea nanoparticles for enhanced anti-tumor activities and hepatoprotectionsynthesis, characterization and in vitro evaluation. J Nutr Biochem. 2015;26(11):1283-1297. doi:10.1016/j.jnutbio.2015.06.003

85. Lee KD, Nagajyothi PC, Sreekanth TVM, Park S. Eco-friendly synthesis of gold nanoparticles (AuNPs) using Inonotus obliquus and their antibacterial, antioxidant and cytotoxic activities. $J$ Ind Eng Chem. 2015;26:67-72. doi:10.1016/j.jiec.2014.11.016

86. Balasubramani G, Ramkumar R, Krishnaveni N, et al. Structural characterization, antioxidant and anticancer properties of gold nanoparticles synthesized from leaf extract (decoction) of Antigonon leptopus Hook. \& Arn. J Trace Elem Med Biol. 2015;30:83-89. doi:10.1016/j.jtemb.2014.11.001

87. Varun S, Sellappa S. Enhanced apoptosis in MCF-7 human breast cancer cells by biogenic gold nanoparticles synthesized from Argemone mexicana leaf extract. Int J Pharm Pharm Sci. 2014;6(8):528-531.

88. Murugan M, Anthony KJP, Jeyaraj M, Rathinam NK, Gurunathan S. Biofabrication of gold nanoparticles and its biocompatibility in human breast adenocarcinoma cells (MCF-7). J Ind Eng Chem. 2014;20 (4):1713-1719. doi:10.1016/j.jiec.2013.08.021

89. Muniyappan N, Nagarajan NS. Green synthesis of gold nanoparticles using Curcuma pseudomontana essential oil, its biological activity and cytotoxicity against human ductal breast carcinoma cells T47D. J Environ Chem Eng. 2014;2(4):2037-2044. doi:10.1016/j.jece.2014.03.004

90. Krishnaraj C, Muthukumaran P, Ramachandran R, Balakumaran MD, Kalaichelvan PT. Acalypha indica Linn: biogenic synthesis of silver and gold nanoparticles and their cytotoxic effects against MDA-MB-231, human breast cancer cells. Biotechnol Rep. 2014;4:42-49. doi:10.1016/j.btre.2014.08.002

91. Gurunathan S, Han J, Park JH, Kim JH. A green chemistry approach for synthesizing biocompatible gold nanoparticles. Nanoscale Res Lett. 2014;9(1):248. doi:10.1186/1556-276X-9-248

92. Fazal S, Jayasree A, Sasidharan S, Koyakutty M, Nair SV, Menon D. Green synthesis of anisotropic gold nanoparticles for photothermal therapy of cancer. ACS Appl Mater Interfaces. 2014;6(11):8080-8089. doi:10.1021/am500302t
93. Ganeshkumar M, Sathishkumar M, Ponrasu T, Dinesh MG, Suguna L. Spontaneous ultra fast synthesis of gold nanoparticles using Punica granatum for cancer targeted drug delivery. Colloids Surf B Biointerfaces. 2013;106:208-216. doi:10.1016/j. colsurfb.2013.01.035

94. Bhat R, Sharanabasava VG, Deshpande R, Shetti U, Sanjeev G, Venkataraman A. Photo-bio-synthesis of irregular shaped functionalized gold nanoparticles using edible mushroom Pleurotus florida and its anticancer evaluation. J Photochem Photobiol B. 2013;125:63-69. doi:10.1016/j.jphotobiol.2013.05.002

95. Punuri JB, Sharma P, Sibyala S, Tamuli R, Bora U. Piper betle-mediated green synthesis of biocompatible gold nanoparticles. Int Nano Lett. 2012;2(1):18. doi:10.1186/2228-5326-2-18

96. Mukherjee S, Sushma V, Patra S, et al. Green chemistry approach for the synthesis and stabilization of biocompatible gold nanoparticles and their potential applications in cancer therapy. Nanotechnology. 2012;23(45):455103. doi:10.1088/0957-4484/ 23/45/455103

97. Babu PJ, Sharma P, Kalita MC, Bora U. Green synthesis of biocompatible gold nanoparticles using Fagopyrum esculentum leaf extract. Front Mater Sci. 2011;5(4):379-387. doi:10.1007/ s11706-011-0153-1

98. Amarnath K, Mathew NL, Nellore J, Siddarth CRV, Kumar J. Facile synthesis of biocompatible gold nanoparticles from Vites vinefera and its cellular internalization against HBL-100 cells. Cancer Nanotechnol. 2011;2(1-6):121-132. doi:10.1007/s12645011-0022-8

99. Nune SK, Chanda N, Shukla R, et al. Green nanotechnology from tea: phytochemicals in tea as building blocks for production of biocompatible gold nanoparticles. J Mater Chem. 2009;19 (19):2912-2920. doi:10.1039/b822015h

100. He C, Lu J, Lin W. Hybrid nanoparticles for combination therapy of cancer. J Controlled Release. 2015;219:224-236. doi:10.1016/ j.jconrel.2015.09.029

101. Ghosh P, Han G, De M, Kim CK, Rotello VM. Gold nanoparticles in delivery applications. Adv Drug Delivery Rev. 2008;60 (11):1307-1315. doi:10.1016/j.addr.2008.03.016

102. Seo JM, Kim EB, Hyun MS, Kim BB, Park TJ. Self-assembly of biogenic gold nanoparticles and their use to enhance drug delivery into cells. Colloids Surf B Biointerfaces. 2015;135:27-34. doi:10.1016/j.colsurfb.2015.07.022

103. Chen X, Han W, Zhao X, Tang W, Wang F. Epirubicin-loaded marine carrageenan oligosaccharide capped gold nanoparticle system for pH-triggered anticancer drug release. Sci Rep. 2019;9 (1):6754. doi:10.1038/s41598-019-43106-9

104. Nguyen VH, Lee B-J. Protein corona: a new approach for nanomedicine design. Int $J$ Nanomedicine. 2017;12:3137-3151. doi:10.2147/IJN.S129300

105. Gorshkov V, Bubis JA, Solovyeva EM, Gorshkov MV, Kjeldsen F. Protein corona formed on silver nanoparticles in blood plasma is highly selective and resistant to physicochemical changes of the solution. Environ Sci. 2019;6(4):1089-1098. doi:10.1039/c8en01054d

106. Zanganeh S, Spitler R, Erfanzadeh M, Alkilany AM, Mahmoudi M. Protein corona: opportunities and challenges. Int $J$ Biochem Cell Biol. 2016;75:143-147. doi:10.1016/j. biocel.2016.01.005

107. Obst K, Yealland G, Balzus B, et al. Protein corona formation on colloidal polymeric nanoparticles and polymeric nanogels: impact on cellular uptake, toxicity, immunogenicity, and drug release properties. Biomacromolecules. 2017;18(6):1762-1771. doi:10.10 21/acs.biomac.7b00158

108. Singh AV, Jahnke T, Wang S, et al. Anisotropic gold nanostructures: optimization via in silico modeling for hyperthermia. ACS Appl Nano Mater. 2018;1(11):6205-6216. doi:10.1021/acsan $\mathrm{m} .8 \mathrm{~b} 01406$ 
109. Singh AV, Alapan Y, Jahnke T, et al. Seed-mediated synthesis of plasmonic gold nanoribbons using cancer cells for hyperthermia applications. $J$ Mater Chem B. 2018;6(46):7573-7581. doi:10.1039/C8TB02239A
110. Singh AV, Jahnke T, Kishore V, et al. Cancer cells biomineralize ionic gold into nanoparticles-microplates via secreting defense proteins with specific gold-binding peptides. Acta Biomater. 2018;71:61-71. doi:10.1016/j.actbio.2018.02.022

\section{Publish your work in this journal}

The International Journal of Nanomedicine is an international, peerreviewed journal focusing on the application of nanotechnology in diagnostics, therapeutics, and drug delivery systems throughout the biomedical field. This journal is indexed on PubMed Central, MedLine, CAS, SciSearch ${ }^{\mathbb{B}}$, Current Contents ${ }^{\mathbb{B}} /$ Clinical Medicine, $^{2}$
Journal Citation Reports/Science Edition, EMBase, Scopus and the Elsevier Bibliographic databases. The manuscript management system is completely online and includes a very quick and fair peer-review system, which is all easy to use. Visit http://www.dovepress.com/ testimonials.php to read real quotes from published authors. 